\title{
EXPONENTIAL ATTRACTOR FOR THE VISCOELASTIC WAVE MODEL WITH TIME-DEPENDENT MEMORY KERNELS
}

\author{
YANAN LI ${ }^{1}$, ZHIJIAN YANG ${ }^{2, *}$ \\ ${ }^{1}$ COLLEGE OF MATHEMATICAL SCIENCES, HARBIN ENGINEERING UNIVERSITY, 150001, CHINA \\ ${ }^{2}$ SCHOOL OF MATHEMATICS AND STATISTICS, ZHENGZHOU UNIVERSITY, 450001, CHINA
}

\begin{abstract}
The paper is concerned with the exponential attractors for the viscoelastic wave model in $\Omega \subset \mathbb{R}^{3}$ :

$$
u_{t t}-h_{t}(0) \Delta u-\int_{0}^{\infty} \partial_{s} h_{t}(s) \Delta u(t-s) \mathrm{d} s+f(u)=h,
$$

with time-dependent memory kernel $h_{t}(\cdot)$ which is used to model aging phenomena of the material. Conti et al $[7,8]$ recently provided the correct mathematical setting for the model and a well-posedness result within the novel theory of dynamical systems acting on time-dependent spaces, recently established by Conti, Pata and Temam [6], and proved the existence and the regularity of the time-dependent global attractor. In this work, we further study the existence of the time-dependent exponential attractors as well as their regularity. We establish an abstract existence criterion via quasi-stability method introduced originally by Chueshov and Lasiecka [2], and on the basis of the theory and technique developed in [7, 8] we further provide a new method to overcome the difficulty of the lack of further regularity to show the existence of the time-dependent exponential attractor. And these techniques can be used to tackle other hyperbolic models.
\end{abstract}

Keywords: Viscoelastic wave model; time-dependent memory kernel; exponential attractors; timedependent phase spaces; longtime behavior of solutions.

2020 Mathematics Subject Classification: 37L30, 37L45, 35B40, 35B41, 35L10.

\section{INTRODUCTION}

In this paper, we investigate the existence of the exponential attractors for the following viscoelastic wave model with time-dependent memory kernel

$$
u_{t t}-h_{t}(0) \Delta u-\int_{0}^{\infty} \partial_{s} h_{t}(s) \Delta u(t-s) \mathrm{d} s+f(u)=h \text { in } \Omega \times(\tau,+\infty),
$$

where $\Omega \subset \mathbb{R}^{3}$ is a bounded domian with the smooth boundary $\partial \Omega$, the time-dependent function

$$
h_{t}(s)=k_{t}(s)+k_{\infty}, \quad k_{\infty}>0, u(\tau-s)=\phi_{\tau}(s), s>0,
$$

SUPPORTED BY NATIONAL NATURAL SCIENCE FOUNDATION OF CHINA (NO.11671367). *CORRESPONDING AUTHOR: ZHIJIAN YANG, E-MAIL: LIYN@HRBEU.EDU.CN (Y. LI), YZJZZUT@TOM.COM (Z. YANG) 
and where $k_{t}(\cdot)$ is convex and summable for every fixed $t, \phi_{\tau}(s)$ is an assigned functions, together with the boundary and initial conditions

$$
\left.u\right|_{\partial \Omega}=0, \quad\left(u(\tau), u_{t}(\tau)\right)=\left(u_{\tau}, v_{\tau}\right) .
$$

Model (1.1) arising from the theory of viscoelasticity was proposed by Conti, Danese, Giorgi and Pata [7] to describe the dynamics of aging materials because the memory kernel $h_{t}(\cdot)$ depends on time, and this feature allows to describe viscoelastic materials whose structural properties evolve over time, say, materials that undergo an aging process which can be reasonably depicted as a loss of the elastic response (for more details, one can see $[5,17,24]$ and references therein). This translates into the study of dynamical systems acting on time-dependent spaces, according to the newly established theory by Conti, Pata and Temam[6], whose inspiration is on the basis of [1,23].

The presence of a time-dependent kernel introduces essential difficulties in the analysis. When the memory kernels are independent $t$, the classical method introduced by Dafermos [10,11] is adding a new variable $\eta$ which is generated by the right-translation semigroup acting on the history space and satisfies a specific differential equation. Under this circumstance, there exist extensive researches on the well-posedness and the longtime behavior of Eq. (1.1), with $h_{t}(s) \equiv h(s)$, see $[9,10,16,18-20,22]$ and references therein. However, this approach become useless for the models with time-dependent memory kernel because the phase spaces for the past history are time-dependent, which causes some problems even in the definition of the time derivative $\partial_{t} \eta$. Hence it is necessary to give a new definition and construct some new estimates about variable $\eta$.

Conti, Danese, Giorgi and Pata [7] introduce the time-dependent memory kernel

$$
\mu_{t}(s):=-\partial_{s} k_{t}(s)=-\partial_{s} h_{t}(s),
$$

and for simplicity let $k_{\infty}=1, \eta_{\tau}(s)=u_{\tau}-\phi_{\tau}(s), s \in \mathbb{R}^{+}$. Then a simple calculation shows that problem (1.1)-(1.3) reads

$$
\partial_{t t} u+A u+\int_{0}^{\infty} \mu_{t}(s) A \eta^{t}(s) \mathrm{d} s+f(u)=h,
$$

where $A$ is the Laplacian with the Dirichlet boundary condition, with domain $D(A)=H^{2}(\Omega) \cap H_{0}^{1}(\Omega)$, and

$$
\eta^{t}(s)=\left\{\begin{array}{l}
u(t)-u(t-s), s \leq t-\tau, \\
\eta_{\tau}(s-t+\tau)+u(t)-u_{\tau}, s>t-\tau,
\end{array}\right.
$$

with the initial condition

$$
\left(u(\tau), \partial_{t} u(\tau), \eta^{\tau}\right)=\left(u_{\tau}, v_{\tau}, \eta_{\tau}\right) .
$$

By proposing a different notion of weak solution where the supplementary differential equation ruling the evolution of $\eta$ is replaced by (1.5), and establishing a family of integral inequalities rather than differential ones as before, Conti, Danese, Giorgi and Pata [7] first provided a global well-posedness result for problem (1.4)-(1.6). Then Conti, Giorgi and Pata [8] further focused on the asymptotic behavior of the weak solutions and proved the existence and the regularity of the time-dependent global attractor. 
EXPONENTIAL ATTRACTOR FOR THE VISCOELASTIC WAVE MODEL WITH TIME-DEPENDENT MEMORY KERNELS ${ }^{1} 3$

The authors $[7,8]$ developed the theory, along with the techniques in their works, and open the way to the longterm analysis of the solutions for the related model with time-dependent memory kernel.

We mention that when the memory kernel $h_{t}(s) \equiv h(s)$ (independent of $t$ ), Danese, Geredeli and Pata [9] have proved the existence of exponential attractors for Eq. (1.1) by using the abstract criterion given in their paper. While the concept of exponential attractors was firstly introduced by Eden et al [13] in the Hilbert space (and later in the Banach space (cf. [14])), which have the advantage of being more stable than global attractors because they have finite fractal dimension and attract trajectories at an exponential rate (cf. [15, 21] for a detail discussion).

However, to the best of the authors' knowledge, there are no results on the exponential attractors for viscoelastic wave model (1.1) (or (1.4)) because of the absence of the exponential attractor theory in the time-dependent phase spaces and the technical difficulties arising from this kind of hyperbolic problem.

The purpose of this paper is to probe this question, and the motivation of this research comes from literatures $[7,8]$. For convenience, we use the same terminology used in [7, 8]. The main strategies can be summarized as follows:

(i) We first give a proper notion of the time-dependent exponential attractors for the dynamical process acting on the time-dependent phase spaces, and provide an abstract existence criterion via quasistability method introduced originally by Chueshov and Lasiecka [2-4]. This criterion can be seen as an extension of that in [26], which provided an abstract result for the existence of the pullback exponential attractors.

(ii) We provide a new method to construct a special attracting family (rather than usual absorbing family) with higher regularity and forward invariance, and based on them to apply the abstract criterion to problem (1.4)-(1.6) to prove the existence and the regularity of the desired time-dependent exponential attractors.

It is worth mentioning that the existence of the time-dependent exponential attractor implies that the fractal dimension of the sections of time-dependent global attractor given by [8] are uniformly bounded, and the application of the abstract criterion is challenging because of the hyperbolicity of Eq. (1.1) (or (1.4)), which leads to non-further regularity of the solutions.

The main contributions of the current paper are that we provide a new method based on the compact attracting family to overcome the difficulty of the lack of further regularity, and to apply the abstract criterion established in this paper (See Theorem 2.5 as well as Corollary 2.6 and Corollary 2.7) to establish the existence of the time-dependent exponential attractor of problem (1.4)-(1.6) (see Theorem 3.5). And this technique can be exported to tackle other hyperbolic models.

The paper is organized as follows. In Section 2, we give the definition of the time-dependent exponential attractors and discuss their existence criterion at an abstract level. In Section 3, we quote the assumptions which are same with those in [7, 8], and state the main theorem of the paper. In Section 4, we first quote some known results coming from literature [7, 8], then based on them we further establish some new estimates which will play key roles for our proving the main theorem. In Section 5, we give the proof of the main theorem. 


\section{TIME-DEPENDENT EXPONENTIAL ATTRACTORS}

In this section, we first quote some notions of the time-dependent global attractor and the related results (cf. [6, 8]), then give the definition of the time-dependent exponential attractor as well as an abstract crirerion on its existence.

Definition 2.1. A two-parameter family of operators $\left\{U(t, \tau): X_{\tau} \rightarrow X_{t} \mid t \geq \tau, \tau \in \mathbb{R}\right\}$ is called a process acting on time-dependent Banach spaces $\left\{X_{t}\right\}_{t \in \mathbb{R}}$ if (i) $U(\tau, \tau)$ is the identity map on $X_{\tau}$; (ii) $U(t, s) U(s, \tau)=U(t, \tau)$ for all $t \geq s \geq \tau$.

Let $U(t, \tau)$ be a process acting on time-dependent Banach spaces $\left\{X_{t}\right\}_{t \in \mathbb{R}}$.

Definition 2.2. A family $\mathcal{B}=\{B(t)\}_{t \in \mathbb{R}}$ is called a uniformly time-dependent absorbing set of the process $U(t, \tau)$ if it is uniformly bounded, i.e.,

$$
\sup _{t \in \mathbb{R}}\|B(t)\|_{X_{t}}:=\sup _{t \in \mathbb{R}} \sup _{\xi \in B(t)}\|\xi\|_{X_{t}}<+\infty,
$$

and for every $R>0$, there exists a $\tau_{e}=\tau_{e}(R) \geq 0$ such that

$$
U(t, \tau) \mathbb{B}_{\tau}(R) \subset B(t) \text { as } t-\tau \geq \tau_{e},
$$

where and in the following $\mathbb{B}_{\tau}(R)=\left\{\xi \in X_{\tau} \mid\|\xi\|_{X_{\tau}} \leq R\right\}$.

Definition 2.3. [6] A family $\mathcal{A}=\{A(t)\}_{t \in \mathbb{R}}$ is called the time-dependent global attractor of the process $U(t, \tau)$ if

: (i) $A(t)$ is compact in $X_{t}$ for each $t \in \mathbb{R}$;

: (ii) $\mathcal{A}$ is pullback attracting, i.e., $\mathcal{A}$ is uniformly bounded and for every uniformly bounded family $\mathcal{D}=\{D(t)\}_{t \in \mathbb{R}}$,

$$
\lim _{\tau \rightarrow-\infty} \operatorname{dist}_{X_{t}}(U(t, \tau) D(\tau), A(t))=0, \quad \forall t \in \mathbb{R},
$$

where

$$
\operatorname{dist}_{X_{t}}(A, B)=\sup _{x \in A} \inf _{y \in B}\|x-y\|_{X_{t}}
$$

is the Hausdorff semidistance of the nonempty sets $A, B \subset X_{t}$.

: (iii) $\mathcal{A}$ is the smallest family with above mentioned properties (i) and (ii), i.e., if a family $\mathcal{A}_{1}=$ $\left\{A_{1}(t)\right\}_{t \in \mathbb{R}}$ is of properties (i) and (ii), then $A(t) \subset A_{1}(t)$ for all $t \in \mathbb{R}$.

Now, we define the time-dependent exponential attractor, which is a generalization of the concept of the pullback exponential attractor, and give its existence criterion.

Definition 2.4. A uniformly bounded family $\mathcal{E}=\{E(t)\}_{t \in \mathbb{R}}$ is called a time-dependent exponential attractor of the process $U(t, \tau)$ if

: (i) $E(t)$ is compact in $X_{t}$ for each $t \in \mathbb{R}$, and its fractal dimension in $X_{t}$ is uniformly bounded, i.e.,

$$
\sup _{t \in \mathbb{R}} \operatorname{dim}_{f}\left(E(t) ; X_{t}\right)<+\infty .
$$

: (ii) $\mathcal{E}$ is semi-invariant, i.e., $U(t, \tau) E(\tau) \subset E(t)$ for all $t \geq \tau$. 
EXPONENTIAL ATTRACTOR FOR THE VISCOELASTIC WAVE MODEL WITH TIME-DEPENDENT MEMORY KERNELS 25

: (iii) There exists a positive constant $\beta$ such that for every uniformly bounded family $\mathcal{D}=\{D(t)\}_{t \in \mathbb{R}}$

$$
\operatorname{dist}_{X_{t}}(U(t, t-\tau) D(t-\tau), E(t)) \leq C(\mathcal{D}) e^{-\beta \tau}, \forall \tau \geq \tau(\mathcal{D}), t \in \mathbb{R},
$$

where $C(\mathcal{D}), \tau(\mathcal{D})$ are positive constants depending only on $\mathcal{D}$.

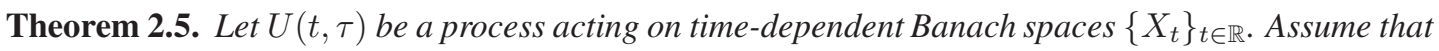
there exist a uniformly bounded family $\mathcal{B}=\{B(t)\}_{t \in \mathbb{R}}$ and a positive constant $T$ such that

$\left(H_{1}\right): B(t)$ is closed in $X_{t}$ for each $t \in \mathbb{R}$, and

$$
U(t, t-\tau) B(t-\tau) \subset B(t), \quad \forall \tau \geq T
$$

$\left(H_{2}\right)$ : there exists a uniform Lipschitz constant $L_{1}>0$ such that

$$
\|U(t, t-\tau) x-U(t, t-\tau) y\|_{X_{t}} \leq L_{1}\|x-y\|_{X_{t-\tau}}
$$

for all $x, y \in B(t-\tau), \tau \in[0, T]$ and $t \in \mathbb{R}$;

$\left(H_{3}\right)$ : there exist a Banach space $Z$ with compact seminorm $n_{Z}(\cdot)$, and a mapping $K_{t}: B(t-T) \rightarrow Z$ for each $t \in \mathbb{R}$ such that for any $x, y \in B(t-T)$,

$$
\begin{aligned}
& \left\|K_{t} x-K_{t} y\right\|_{Z} \leq L\|x-y\|_{X_{t-T}}, \\
& \|U(t, t-T) x-U(t, t-T) y\|_{X_{t}} \leq \eta\|x-y\|_{X_{t-T}}+n_{Z}\left(K_{t} x-K_{t} y\right),
\end{aligned}
$$

where $\eta \in(0,1 / 2), L>0$ are constants independent of $t$.

Then, there exists a semi-invariant family $\mathcal{E}=\{E(t)\}_{t \in \mathbb{R}}$ possessing the following properties:

: (i) the section $E(t) \subset B(t)$ is compact in $X_{t}$ for each $t \in \mathbb{R}$ and its fractal dimension in $X_{t}$ is uniformly bounded, i.e.,

$$
\sup _{t \in \mathbb{R}} \operatorname{dim}_{f}\left(E(t) ; X_{t}\right) \leq\left[\ln \left(\frac{1}{2 \eta}\right)\right]^{-1} \ln m_{Z}\left(\frac{2 L}{\eta}\right)<+\infty,
$$

where $m_{Z}(R)$ is the maximal number of elements $z_{i}$ in the ball $\left\{z \in Z \mid\|z\|_{Z} \leq R\right\}$ such that $n_{Z}\left(z_{i}-z_{j}\right)>1, i \neq j$;

: (ii) there exist positive constants $\beta, C$ and $\tau_{0}$ such that

$$
\operatorname{dist}_{X_{t}}(U(t, t-\tau) B(t-\tau), E(t)) \leq C e^{-\beta \tau} \text { as } \tau \geq \tau_{0}, \forall t \in \mathbb{R} .
$$

Corollary 2.6. Let the assumptions of Theorem 2.5 be valid. If the family $\mathcal{B}=\{B(t)\}_{t \in \mathbb{R}}$ is a uniformly time-dependent absorbing set of the process $U(t, \tau)$, then the family $\mathcal{E}=\{E(t)\}_{t \in \mathbb{R}}$ given in Theorem 2.5 is a time-dependent exponential attractor of the process $U(t, \tau)$.

Proof. For any uniformly bounded family $\mathcal{D}=\{D(t)\}_{t \in \mathbb{R}}$, there exist positive constants $R$ and $e(R)$ such that $D(t) \subset \mathbb{B}_{t}(R)$ for all $t \in \mathbb{R}$ and

$$
U(t, t-\tau) D(t-\tau) \subset U(t, t-\tau) \mathbb{B}_{t-\tau}(R) \subset B(t), \quad \forall \tau \geq e(R)
$$


Thus, it follows from (2.6) that, for every $t \in \mathbb{R}, \tau \geq e(R)+\tau_{0}$,

$$
\begin{aligned}
& \operatorname{dist}_{X_{t}}(U(t, t-\tau) D(t-\tau), E(t)) \\
\leq & \operatorname{dist}_{X_{t}}(U(t, t-\tau+e(R)) U(t-\tau+e(R), t-\tau) D(t-\tau), E(t)) \\
\leq & \operatorname{dist}_{X_{t}}(U(t, t-\tau+e(R)) B(t-\tau+e(R)), E(t)) \\
\leq & C e^{\beta e(R)} e^{-\beta \tau} .
\end{aligned}
$$

This completes the proof.

Corollary 2.7. Let the assumptions of Theorem 2.5 be valid. If the process $U(t, \tau)$ possesses a uniformly time-dependent absorbing set $\left\{\mathbb{B}_{t}\left(R_{1}\right)\right\}_{t \in \mathbb{R}}$ possessing the following properties:

(i) there is a positive constant $R_{2}>R_{1}$ such that $B(t) \subset \mathbb{B}_{t}\left(R_{2}\right)$ for all $t \in \mathbb{R}$ and formula (2.2) holds on $\mathbb{B}_{t}\left(R_{2}\right)$, that is

$$
\|U(t, t-\tau) x-U(t, t-\tau) y\|_{X_{t}} \leq L_{1}\|x-y\|_{X_{t-\tau}}
$$

for all $x, y \in \mathbb{B}_{t-\tau}\left(R_{2}\right), \tau \in[0, T]$ and $t \in \mathbb{R}$;

(ii) there exist positive constants $\kappa, \tau_{1}$ and $C_{0}$ such that

$$
\operatorname{dist}_{X_{t}}\left(U(t, t-\tau) \mathbb{B}_{t-\tau}\left(R_{1}\right), B(t)\right) \leq C_{0} e^{-\kappa \tau}, \quad \forall t \in \mathbb{R}, \tau \geq \tau_{1} .
$$

Then the family $\mathcal{E}=\{E(t)\}_{t \in \mathbb{R}}$ given in Theorem 2.5 is a time-dependent exponential attractor of the process $U(t, \tau)$.

Proof. By the definition of uniformly time-dependent absorbing set, there exists a positive constant $e\left(R_{1}\right)>\tau_{1}$ such that for any given $\theta \in(0,1)$,

$$
U(t-\tau \theta, t-\tau) \mathbb{B}_{t-\tau}\left(R_{1}\right) \subset \mathbb{B}_{t-\tau \theta}\left(R_{1}\right), \quad \forall \tau \geq(1-\theta)^{-1} e\left(R_{1}\right), t \in \mathbb{R} .
$$

Thus, it follows from (2.6)-(2.7) and the fact: $B(t) \subset \mathbb{B}_{t}\left(R_{2}\right)$ for all $t \in \mathbb{R}$ that

$$
\begin{aligned}
& \operatorname{dist}_{X_{t}}\left(U(t, t-\tau) \mathbb{B}_{t-\tau}\left(R_{1}\right), E(t)\right) \\
\leq & \operatorname{dist}_{X_{t}}\left(U(t, t-\tau \theta) U(t-\tau \theta, t-\tau) \mathbb{B}_{t-\tau}\left(R_{1}\right), U(t, t-\tau \theta) B(t-\tau \theta)\right) \\
& +\operatorname{dist}_{X_{t}}(U(t, t-\tau \theta) B(t-\tau \theta), E(t)) \\
\leq & L_{1}^{\left(\frac{\tau \theta}{T}+1\right)} \operatorname{dist}_{X_{t-\tau \theta}}\left(U(t-\tau \theta, t-\tau) \mathbb{B}_{t-\tau}\left(R_{1}\right), B(t-\tau \theta)\right)+C e^{-\beta \tau \theta} \\
\leq & C_{0} L_{1} e^{\left(\frac{\theta}{T} \ln L_{1}+\kappa \theta-\kappa\right) \tau}+C e^{-\beta \tau \theta}=\left(C+C_{0} L_{1}\right) e^{-\beta^{\prime} \tau}
\end{aligned}
$$

for all $\tau \geq \tau_{2}:=\max \left\{\theta^{-1} \tau_{0},(1-\theta)^{-1} e\left(R_{1}\right)\right\}$ and $t \in \mathbb{R}$, where

$$
\theta=\frac{T \kappa}{2\left(\ln L_{1}+T \kappa\right)} \in(0,1) \text { and } \beta^{\prime}=\min \left\{\frac{\kappa}{2}, \frac{T \kappa \beta}{2\left(\ln L_{1}+T \kappa\right)}\right\}>0 .
$$

Then, repeating the same argument as the proof of Corollary 2.6 and using estimate (2.9), we obtain that for any uniformly bounded family $\mathcal{D}=\{D(t)\}_{t \in \mathbb{R}}$,

$$
\operatorname{dist}_{X_{t}}(U(t, t-\tau) D(t-\tau), E(t)) \leq\left(C+C_{0} L_{1}\right) e^{\beta^{\prime} e(R)} e^{-\beta^{\prime} \tau}, \quad \forall \tau \geq \tau_{2}+e(R), t \in \mathbb{R} .
$$

This completes the proof. 
EXPONENTIAL ATTRACTOR FOR THE VISCOELASTIC WAVE MODEL WITH TIME-DEPENDENT MEMORY KERNELS ${ }^{3} 7$

Proof of Theorem 2.5. For clarity and without loss of generality, we assume $T=1$. Since $\mathcal{B}=$ $\{B(t)\}_{t \in \mathbb{R}}$ is a uniformly bounded family, we have

$$
B(t) \subset \mathbb{B}_{t}\left(R_{0}\right), \quad \forall t \in \mathbb{R}
$$

for some positive constant $R_{0}$. Thus $N_{t}\left(B(t), R_{0}\right)=1$ for all $t \in \mathbb{R}$, here $N_{t}(B, \epsilon)$ denotes the cardinality of minimal covering of the set $B \subset X_{t}$ by its closed subsets of diameter $\leq 2 \epsilon$.

Conditions $\left(H_{1}\right)-\left(H_{3}\right)$ show that

$$
U(m, n) B(n) \subset B(m), \quad \forall m \geq n,
$$

and for all $x, y \in B(n-1)$ and $n \in \mathbb{Z}$,

$$
\begin{aligned}
& \|U(n, n-1) x-U(n, n-1) y\|_{X_{n}} \leq L_{1}\|x-y\|_{X_{n-1}}, \\
& \left\|K_{n} x-K_{n} y\right\|_{Z} \leq L\|x-y\|_{X_{n-1}}, \\
& \|U(n, n-1) x-U(n, n-1) y\|_{X_{n}} \leq \eta\|x-y\|_{X_{n-1}}+n_{Z}\left(K_{n} x-K_{n} y\right) .
\end{aligned}
$$

Now we show the following formula for all $n \in \mathbb{Z}$ by induction on $k \in \mathbb{N}$

$$
N_{n}(k):=N_{n}\left(U(n, n-k) B(n-k),(2 \eta)^{k} R_{0}\right) \leq\left[m_{Z}\left(\frac{2 L}{\eta}\right)\right]^{k} .
$$

Let, for $k=1$ and $n \in \mathbb{Z}$,

$$
n(1):=\aleph\left\{x_{l} \in B(n-1) \mid n_{Z}\left(K_{n} x_{j}-K_{n} x_{i}\right)>\eta R_{0}, j \neq i\right\},
$$

where $\aleph\{\cdots\}$ denotes the maximal number of elements with the given properties. And let

$$
\mathcal{B}(n-1)=K_{n} B(n-1)=\left\{K_{n} x \mid x \in B(n-1)\right\} .
$$

Formula (2.12) implies that

$$
\begin{aligned}
& \operatorname{diam}(\mathcal{B}(n-1) ; Z) \leq L \operatorname{diam}\left(B(n-1) ; X_{n-1}\right) \leq 2 L R_{0}, \\
& \mathcal{B}(n-1) \subset \mathbb{B}_{Z}\left(y ; 2 L R_{0}\right)=\left\{z \in Z \mid\|z-y\|_{Z} \leq 2 L R_{0}\right\} \text { for some } y \in Z .
\end{aligned}
$$

By the linearity and compactness of the seminorm $n_{Z}(\cdot)$,

$$
\begin{aligned}
n(1) & =\aleph\left\{z_{l} \in \mathcal{B}(n-1) \mid n_{Z}\left(z_{j}-z_{i}\right)>\eta R_{0}, j \neq i\right\} \\
& \leq \aleph\left\{z_{l} \in \mathbb{B}_{Z}\left(y ; 2 L R_{0}\right) \mid n_{Z}\left(z_{j}-z_{i}\right)>\eta R_{0}, j \neq i\right\} \\
& =\aleph\left\{z_{l} \in \mathbb{B}_{Z}\left(0 ; \frac{2 L}{\eta}\right) \mid n_{Z}\left(z_{j}-z_{i}\right)>1, j \neq i\right\} \\
& =m_{Z}\left(\frac{2 L}{\eta}\right)<+\infty .
\end{aligned}
$$

Consequently, there exists a maximal subset $\left\{x_{j}\right\}_{j=1}^{n(1)}$ of $B(n-1)$ such that

$$
n_{Z}\left(K_{n} x_{j}-K_{n} x_{l}\right)>\eta R_{0}, \quad j \neq l,
$$


and

$$
\begin{aligned}
& B(n-1)=\bigcup_{j=1}^{n(1)} C_{j} \text { with } C_{j}=\left\{x \in B(n-1) \mid n_{Z}\left(K_{n} x-K_{n} x_{j}\right) \leq \eta R_{0}\right\}, \\
& U(n, n-1) B(n-1)=\bigcup_{j=1}^{n(1)} U(n, n-1) C_{j} .
\end{aligned}
$$

Formula (2.13) implies that, for all $x, y \in C_{j}$ and all $j=1, \cdots, n(1)$,

$$
\begin{aligned}
& \|U(n, n-1) x-U(n, n-1) y\|_{X_{n}} \\
\leq & \eta\|x-y\|_{X_{n-1}}+n_{Z}\left(K_{n} x-K_{n} y\right) \\
\leq & \eta \operatorname{diam}\left(B(n-1) ; X_{n-1}\right)+2 \eta R_{0} \leq 4 \eta R_{0},
\end{aligned}
$$

which implies

$$
\operatorname{diam}\left(U(n, n-1) C_{j} ; X_{n}\right) \leq 4 \eta R_{0}, \quad j=1, \cdots, n(1) .
$$

Thus, the combination of (2.15) and (2.16) shows that

$$
N_{n}(1)=N_{n}\left(U(n, n-1) B(n-1), 2 \eta R_{0}\right) \leq m_{Z}\left(\frac{2 L}{\eta}\right),
$$

that is, formula (2.14) holds for $k=1$ and $n \in \mathbb{Z}$.

Assume that formula (2.14) holds for all $1 \leq k \leq k_{0}$ and $n \in \mathbb{Z}$. We prove that it also holds for $k=k_{0}+1$. Due to

$$
U\left(n, n-k_{0}-1\right) B\left(n-k_{0}-1\right)=U(n, n-1) U\left(n-1, n-k_{0}-1\right) B\left(n-k_{0}-1\right),
$$

and

$$
N_{n}\left(k_{0}\right)=N_{n}\left(U\left(n, n-k_{0}\right) B\left(n-k_{0}\right),(2 \eta)^{k_{0}} R_{0}\right) \leq\left[m_{Z}\left(\frac{2 L}{\eta}\right)\right]^{k_{0}}, \forall n \in \mathbb{Z}
$$

there exists a minimal covering $\left\{F_{i}\right\}_{i=1}^{N_{n-1}\left(k_{0}\right)}$ of $U\left(n-1, n-k_{0}-1\right) B\left(n-k_{0}-1\right)$ by its closed subsets of diameter $\leq 2(2 \eta)^{k_{0}} R_{0}$, that is,

$$
\begin{aligned}
& \bigcup_{i=1}^{N_{n-1}\left(k_{0}\right)} F_{i}=U\left(n-1, n-k_{0}-1\right) B\left(n-k_{0}-1\right) \subset B(n-1), \\
& \operatorname{diam}\left(F_{i} ; X_{n-1}\right) \leq 2(2 \eta)^{k_{0}} R_{0}, \quad i=1, \cdots, N_{n-1}\left(k_{0}\right) .
\end{aligned}
$$

Let

$$
\mathcal{F}_{i}=K_{n} F_{i}=\left\{K_{n} z \mid z \in F_{i}\right\} \subset Z, \quad i=1, \cdots, N_{n-1}\left(k_{0}\right) .
$$

The combination of (2.12) and (2.19) shows that

$$
\begin{aligned}
& \operatorname{diam}\left(\mathcal{F}_{i} ; Z\right) \leq L \operatorname{diam}\left(F_{i} ; X_{n-1}\right) \leq 2 L(2 \eta)^{k_{0}} R_{0}, \\
& \mathcal{F}_{i} \subset \mathbb{B}_{Z}\left(y_{i} ; 2 L(2 \eta)^{k_{0}} R_{0}\right)=\left\{z \in Z \mid\left\|z-y_{i}\right\|_{Z} \leq 2 L(2 \eta)^{k_{0}} R_{0}\right\} \text { for some } y_{i} \in Z .
\end{aligned}
$$


EXPONENTIAL ATTRACTOR FOR THE VISCOELASTIC WAVE MODEL WITH TIME-DEPENDENT MEMORY KERNELS 49

Hence,

$$
\begin{aligned}
n_{i}\left(k_{0}+1\right): & =\aleph\left\{x_{l} \in F_{i} \mid n_{Z}\left(K_{n} x_{j}-K_{n} x_{m}\right)>\eta(2 \eta)^{k_{0}} R_{0}, j \neq m\right\} \\
& =\aleph\left\{z_{l} \in \mathcal{F}_{i} \mid n_{Z}\left(z_{j}-z_{m}\right)>\eta(2 \eta)^{k_{0}} R_{0}, j \neq m\right\} \\
& \leq \aleph\left\{z_{l} \in \mathbb{B}_{Z}\left(y_{i} ; 2 L(2 \eta)^{k_{0}} R_{0}\right) \mid n_{Z}\left(z_{j}-z_{m}\right)>\eta(2 \eta)^{k_{0}} R_{0}, j \neq m\right\} \\
& =\aleph\left\{z_{l} \in \mathbb{B}_{Z}\left(0 ; \frac{2 L}{\eta}\right) \mid n_{Z}\left(z_{j}-z_{m}\right)>1, j \neq m\right\} \\
& =m_{Z}\left(\frac{2 L}{\eta}\right)<+\infty .
\end{aligned}
$$

Consequently,

$$
F_{i}=\bigcup_{j=1}^{n_{i}\left(k_{0}+1\right)} C_{j}^{i} \text { with } C_{j}^{i}=\left\{x \in F_{i} \mid n_{Z}\left(K_{n} x-K_{n} x_{j}^{i}\right) \leq \eta(2 \eta)^{k_{0}} R_{0}\right\},
$$

where $\left\{x_{j}^{i}\right\}_{j=1}^{n_{i}\left(k_{0}+1\right)}$ is the maximal subset of $F_{i}$ such that

$$
n_{Z}\left(K_{n} x_{l}^{i}-K_{n} x_{j}^{i}\right)>\eta(2 \eta)^{k_{0}} R_{0}, \quad l \neq j .
$$

It follows from (2.13) and (2.17)-(2.21) that

$$
\begin{aligned}
& \operatorname{diam}\left(U(n, n-1) C_{j} ; X_{n}\right) \leq \eta \operatorname{diam}\left(F_{i} ; X_{n-1}\right)+(2 \eta)^{k_{0}+1} R_{0} \leq 2(2 \eta)^{k_{0}+1} R_{0}, \\
& U\left(n, n-k_{0}-1\right) B\left(n-k_{0}-1\right)=\bigcup_{i=1}^{N_{n-1}\left(k_{0}\right)} \bigcup_{j=1}^{n_{i}\left(k_{0}+1\right)} U(n, n-1) C_{j}^{i},
\end{aligned}
$$

which imply that

$$
\begin{aligned}
N_{n}\left(k_{0}+1\right) & =N_{n}\left(U\left(n, n-k_{0}-1\right) B\left(n-k_{0}-1\right),(2 \eta)^{k_{0}+1} R_{0}\right) \\
& \leq \sum_{i=1}^{N_{n-1}\left(k_{0}\right)} n_{i}\left(k_{0}+1\right) \leq\left[m_{Z}\left(\frac{2 L}{\eta}\right)\right]^{k_{0}+1} .
\end{aligned}
$$

Therefore, formula (2.14) is valid.

It follows from (2.10) and (2.14) that, for every $n \in \mathbb{Z}$ and every $k \geq 1$, there is a finite subset $V_{k}(n)$ possessing the following properties:

$$
\begin{aligned}
& \operatorname{Card}\left(V_{k}(n)\right) \leq\left[m_{Z}\left(\frac{2 L}{\eta}\right)\right]^{k}, \\
& V_{k}(n) \subset U(n, n-k) B(n-k) \subset B(n) \subset X_{n}, \\
& U(n, n-k) B(n-k) \subset \bigcup_{h \in V_{k}(n)}\left[\mathbb{B}_{n}\left(2(2 \eta)^{k} R_{0}\right)+\{h\}\right] .
\end{aligned}
$$


For any given $n \in \mathbb{Z}$, we define by induction that

$$
\left\{\begin{array}{l}
E_{1}(n)=V_{1}(n), \\
E_{k}(n)=V_{k}(n) \cup\left[U(n, n-1) E_{k-1}(n-1)\right], k \geq 2, \\
E(n)=\left[\bigcup_{k \geq 1} E_{k}(n)\right]_{X_{n}},
\end{array}\right.
$$

where $[\cdot]_{X_{n}}$ denotes the closure in $X_{n}$. Thus it follows from (2.10) and (2.23)-(2.24) that

$$
\begin{aligned}
& E_{k}(n)=\bigcup_{l=0}^{k-1} U(n, n-l) V_{k-l}(n-l) \subset U(n, n-k) B(n-k), \\
& U(n+1, n) E_{k}(n) \subset E_{k+1}(n+1), \\
& E(n) \subset U(n, n-1) B(n-1) \subset B(n), \quad \forall n \in \mathbb{Z}, k \geq 1 .
\end{aligned}
$$

Moreover, we infer from (2.22) and (2.26) that

$$
\begin{aligned}
\operatorname{Card}\left(E_{k}(n)\right) & \leq \sum_{l=0}^{k-1} \operatorname{Card}\left(V_{k-l}(n-l)\right) \\
& \leq \sum_{l=0}^{k-1}\left[m_{Z}\left(\frac{2 L}{\eta}\right)\right]^{k-l} \leq\left[m_{Z}\left(\frac{2 L}{\eta}\right)\right]^{k+1}, \forall n \in \mathbb{Z}, k \geq 1 .
\end{aligned}
$$

We show that the family $\{E(n)\}_{n \in \mathbb{Z}}$ is of the following properties:

(i) Semi-invariance. By the Lipschitz continuity (2.11), formulas (2.25) and (2.27)-(2.28),

$$
U(n, l) E(l) \subset\left[\bigcup_{k \geq 1} U(n, l) E_{k}(l)\right]_{X_{n}} \subset\left[\bigcup_{k \geq 1} E_{k+n-l}(n)\right]_{X_{n}} \subset E(n), \quad \forall n \geq l .
$$

(ii) Pullback exponential attractiveness. We see from (2.25) that $V_{k}(n) \subset E(n)$ holds for all $n \in \mathbb{Z}$ and $k \geq 1$. Thus we infer from (2.24) that

$$
\begin{aligned}
& \operatorname{dist}_{X_{n}}(U(n, n-k) B(n-k), E(n)) \\
\leq & \operatorname{dist}_{X_{n}}\left(U(n, n-k) B(n-k), V_{k}(n)\right) \\
\leq & 2(2 \eta)^{k} R_{0}, \quad \forall k \geq 1, n \in \mathbb{Z} .
\end{aligned}
$$

(iii) Boundedness of the fractal dimension. For any $\epsilon \in(0,1)$, there is a unique $k_{\epsilon} \in \mathbb{N}^{+}$such that

$$
2(2 \eta)^{k_{\epsilon}} R_{0}<\epsilon \leq 2(2 \eta)^{k_{\epsilon}-1} R_{0} .
$$

Obviously, $k_{\epsilon} \rightarrow \infty$ as $\epsilon \rightarrow 0^{+}$. It follows from (2.10) and (2.26) that

$$
E_{k}(n) \subset U(n, n-k) B(n-k) \subset U\left(n, n-k_{\epsilon}\right) B\left(n-k_{\epsilon}\right), \quad \forall k \geq k_{\epsilon}, n \in \mathbb{Z},
$$

which implies that

$$
E(n) \subset\left(\bigcup_{k<k_{\epsilon}} E_{k}(n)\right) \bigcup\left[U\left(n, n-k_{\epsilon}\right) B\left(n-k_{\epsilon}\right)\right]_{X_{n}}, \quad \forall n \in \mathbb{Z} .
$$


EXPONENTIAL ATTRACTOR FOR THE VISCOELASTIC WAVE MODEL WITH TIME-DEPENDENT MEMORY KERNELS ${ }_{11}$

Thus it follows from (2.14), (2.29) and (2.32) that

$$
\begin{aligned}
N_{n}(E(n), \epsilon) & \leq N_{n}\left(E(n), 2(2 \eta)^{k_{\epsilon}} R_{0}\right) \\
& \leq \sum_{k<k_{\epsilon}} \operatorname{Card}\left(E_{k}(n)\right)+N_{n}\left(U\left(n, n-k_{\epsilon}\right) B\left(n-k_{\epsilon}\right), 2(2 \eta)^{k_{\epsilon}} R_{0}\right) \\
& \leq \sum_{k<k_{\epsilon}}\left[m_{Z}\left(\frac{2 L}{\eta}\right)\right]^{k+1}+N_{n}\left(k_{\epsilon}\right) \leq 2\left[m_{Z}\left(\frac{2 L}{\eta}\right)\right]^{k_{\epsilon}+1}<+\infty .
\end{aligned}
$$

By the arbitrariness of $\epsilon \in(0,1)$, we see from (2.33) that $E(n)$ is a compact subset of $X_{n}$. Moreover, estimates (2.32)-(2.33) and a simple calculation shows that

$$
\frac{\ln N_{n}(E(n), \epsilon)}{\ln (1 / \epsilon)} \leq \frac{\left(k_{\epsilon}+1\right) \ln \left[m_{Z}\left(\frac{2 L}{\eta}\right)\right]+\ln 2}{\left(k_{\epsilon}-1\right) \ln (1 / 2 \eta)-\ln \left(2 R_{0}\right)}, \epsilon \in(0,1)
$$

which implies that

$$
\operatorname{dim}_{f}\left(E(n) ; X_{n}\right)=\limsup _{\epsilon \rightarrow 0^{+}} \frac{\ln N_{n}(E(n), \epsilon)}{\ln (1 / \epsilon)} \leq\left[\ln \left(\frac{1}{2 \eta}\right)\right]^{-1} \ln \left[m_{Z}\left(\frac{2 L}{\eta}\right)\right], \forall n \in \mathbb{Z} .
$$

For any $t \in \mathbb{R}$, there exists a unique $n \in \mathbb{Z}$ such that $t \in[n, n+1)$. Let

$$
E(t)=U(t, n) E(n)
$$

We claim that $\{E(t)\}_{t \in \mathbb{R}}$ is the desired family.

(i) It follows from formulas (2.1) and (2.28) that

$$
E(t)=U(t, n) E(n) \subset U(t, n) U(n, n-1) B(n-1) \subset B(t), \quad \forall t \in \mathbb{R} .
$$

Moreover, by the Lipschitz continuity of $U(t, n): B(n) \subset X_{n} \rightarrow X_{t}$ (see (2.2)), and the compactness of $E(n)$ in $X_{n}$, we know that $E(t)$ is a compact subset of $X_{t}$ and by (2.34),

$$
\operatorname{dim}_{f}\left(E(t) ; X_{t}\right) \leq \operatorname{dim}_{f}\left(E(n) ; X_{n}\right) \leq\left[\ln \left(\frac{1}{2 \eta}\right)\right]^{-1} \ln \left[m_{Z}\left(\frac{2 L}{\eta}\right)\right]
$$

(ii) For any $t \geq s \in \mathbb{R}$, let $t=n+t_{1}, s=m+s_{1}$ for some $n, m \in \mathbb{Z}$ and $t_{1}, s_{1} \in[0,1)$. Then, by formula (2.35) and the semi-invariance of $\{E(n)\}_{n \in \mathbb{Z}}$ (see (2.30)), we have

$$
\begin{aligned}
U(t, s) E(s) & =U(t, n) U(n, s) U(s, m) E(m) \\
& =U(t, n) U(n, m) E(m) \subset U(t, n) E(n)=E(t) .
\end{aligned}
$$

(iii) For any given $t \in \mathbb{R}$ and $\tau \geq 3$, there exist $n \in \mathbb{Z}$ and $k_{\tau} \in \mathbb{N}^{+}$such that

$$
t \in[n, n+1) \text { and } \tau \in\left[k_{\tau}+2, k_{\tau}+3\right)
$$

which imply that

$$
n-k_{\tau}-(t-\tau) \geq 1,-k_{\tau}<-\tau+3
$$


and by formula (2.1),

$$
\begin{aligned}
U(t, t-\tau) B(t-\tau) & =U(t, n) U\left(n, n-k_{\tau}\right) U\left(n-k_{\tau}, t-\tau\right) B(t-\tau) \\
& \subset U(t, n) U\left(n, n-k_{\tau}\right) B\left(n-k_{\tau}\right) .
\end{aligned}
$$

Thus we infer from the Lipschitz continuity (2.2), estimate (2.31) and formulas (2.35)-(2.36) that

$$
\begin{aligned}
& \operatorname{dist}_{X_{t}}(U(t, t-\tau) B(t-\tau), E(t)) \\
\leq & \operatorname{dist}_{X_{t}}\left(U(t, n) U\left(n, n-k_{\tau}\right) B\left(n-k_{\tau}\right), U(t, n) E(n)\right) \\
\leq & L_{1} \operatorname{dist}_{X_{n}}\left(U\left(n, n-k_{\tau}\right) B\left(n-k_{\tau}\right), E(n)\right) \\
\leq & 2 L_{1}(2 \eta)^{k_{\tau}} R_{0}=2 L_{1} R_{0} e^{-\beta k_{\tau}} \leq C e^{-\beta \tau}, \forall t \in \mathbb{R}, \tau \geq 3,
\end{aligned}
$$

with $\beta=\ln \frac{1}{2 \eta}$ and $C=2 L_{1} R_{0} e^{3}$. This completes the proof.

Remark 2.8. Theorem 2.5 and its Corollaries 2.6 and 2.7 are established, for simplicity, in a Banach space framework because of the definition of phase space $\mathcal{H}_{t}$ in the time-dependent memory kernel problem. However, they are still valid if the family of Banach spaces $\left\{X_{t}\right\}_{t \in \mathbb{R}}$ there is replaced by a family of normed linear spaces $\left\{X_{t}\right\}_{t \in \mathbb{R}}$.

\section{Preliminaries And MAin Results on the Model (1.4)-(1.6)}

For any $\sigma \in \mathbb{R}$, we define the compactly nested Hilbert spaces

$$
H^{\sigma}=D\left(A^{\sigma / 2}\right)
$$

endowed with the inner products and the norms:

$$
\langle u, v\rangle_{\sigma}=\left\langle A^{\sigma / 2} u, A^{\sigma / 2} v\right\rangle_{L^{2}},\|u\|_{\sigma}=\left\|A^{\sigma / 2} u\right\|_{L^{2}},
$$

respectively, where and in the context the operator $A$ is as shown in Eq. (1.4), and $L^{2}=L^{2}(\Omega)$. The index $\sigma$ will be omitted whenever zero. The symbol $\langle\cdot, \cdot\rangle$ for the $L^{2}$-inner product will also be used for the duality pairing between the dual spaces. We denote

$$
L^{p}=L^{p}(\Omega), \quad H=L^{2}, \quad H^{1}=H_{0}^{1}(\Omega), H^{-1}=H^{-1}(\Omega), \quad H^{2}=H^{2}(\Omega) \cap H_{0}^{1}(\Omega),
$$

with $p \geq 1$. For every fixed time $t$ and index $\sigma$, we introduce the weighted $L^{2}$-spaces, hereafter they are called memory spaces,

$$
\mathcal{M}_{t}^{\sigma}=L_{\mu_{t}}^{2}\left(\mathbb{R}^{+} ; H^{\sigma+1}\right)=\left\{\xi: \mathbb{R}^{+} \rightarrow H^{\sigma+1} \mid \int_{0}^{\infty} \mu_{t}(s)\|\xi(s)\|_{\sigma+1}^{2} \mathrm{~d} s<\infty\right\}
$$

equipped with the weighted $L^{2}$-inner products

$$
\langle\eta, \xi\rangle_{\mathcal{M}_{t}^{\sigma}}=\int_{0}^{\infty} \mu_{t}(s)\langle\eta(s), \xi(s)\rangle_{\sigma+1} \mathrm{~d} s .
$$

And we define the extended memory spaces

$$
\mathcal{H}_{t}^{\sigma}=H^{\sigma+1} \times H^{\sigma} \times \mathcal{M}_{t}^{\sigma}
$$

equipped with the usual product norm

$$
\|(u, v, \eta)\|_{\mathcal{H}_{t}^{\sigma}}^{2}=\|u\|_{\sigma+1}^{2}+\|v\|_{\sigma}^{2}+\|\eta\|_{\mathcal{M}_{t}^{\sigma}}^{2} .
$$


EXPONENTIAL ATTRACTOR FOR THE VISCOELASTIC WAVE MODEL WITH TIME-DEPENDENT MEMORY KERNELS ${ }_{13}$

For any $r>0$, we denote by

$$
\mathbb{B}_{t}^{\sigma}(r)=\left\{z \in \mathcal{H}_{t}^{\sigma} \mid\|z\|_{\mathcal{H}_{t}^{\sigma}} \leq r\right\}
$$

the closed $r$-ball centered at zero of $\mathcal{H}_{t}^{\sigma}$.

\subsection{Assumptions and well-posedness.}

Assumption 3.1. [8] (i) Let $g \in H$ be independent of time, and let $f \in C^{2}(\mathbb{R})$ with $f(0)=0$,

$$
\left|f^{\prime \prime}(u)\right| \leq c(1+|u|) \text { and } \liminf _{|u| \rightarrow \infty} f^{\prime}(u)>-\lambda_{1},
$$

for some $c \geq 0$, where $\lambda_{1}>0$ is the first eigenvalue of $A$.

(ii) The map $(t, s) \mapsto \mu_{t}(s): \mathbb{R} \times \mathbb{R}^{+} \rightarrow \mathbb{R}^{+}$possesses the following properties:

$\left(M_{1}\right)$ : For every fixed $t \in \mathbb{R}$, the map $s \mapsto \mu_{t}(s)$ is nonincreasing, absolutely continuous and summable. We denote the total mass of $\mu_{t}$ by $\kappa(t)=\int_{0}^{\infty} \mu_{t}(s) \mathrm{d} s$.

$\left(M_{2}\right)$ : For every $\tau \in \mathbb{R}$, there exists a function $K_{\tau}:[\tau, \infty) \rightarrow \mathbb{R}^{+}$, summable on any interval $[\tau, T]$, such that $\mu_{t}(s) \leq K_{\tau}(t) \mu_{\tau}(s)$ for every $t \geq \tau$ and every $s>0$.

$\left(M_{3}\right)$ : For almost every fixed $s>0$, the map $t \mapsto \mu_{t}(s)$ is differentiable for all $t \in \mathbb{R}$, and $(t, s) \mapsto$ $\mu_{t}(s) \in L^{\infty}(\mathcal{K}),(t, s) \mapsto \partial_{t} \mu_{t}(s) \in L^{\infty}(\mathcal{K})$ for every compact set $\mathcal{K} \subset \mathbb{R} \times \mathbb{R}^{+}$.

$\left(M_{4}\right)$ : There exists a $\delta>0$ such that $\partial_{t} \mu_{t}(s)+\partial_{s} \mu_{t}(s)+\delta \kappa(t) \mu_{t}(s) \leq 0$ for every $t \in \mathbb{R}$ and almost every $s>0$.

$\left(M_{5}\right)$ : The function $t \rightarrow \kappa(t)$ fulfills: $\inf _{t \in \mathbb{R}} \kappa(t)>0$.

$\left(M_{6}\right)$ : The function $t \mapsto \partial_{t} \mu_{t}(s)$ satisfies the uniform integral estimate: $\sup _{t \in \mathbb{R}} \frac{1}{[\kappa(t)]^{2}} \int_{0}^{\infty}\left|\partial_{t} \mu_{t}(s)\right| \mathrm{d} s<$ $\infty$.

$\left(M_{7}\right)$ : For every $t \in \mathbb{R}$, the function $s \mapsto \mu_{t}(s)$ is bounded about zero, with $\sup _{t \in \mathbb{R}} \frac{\mu_{t}(0)}{[\kappa(t)]^{2}}<\infty$.

$\left(M_{8}\right)$ : For every $a<b \in \mathbb{R}$, there exists a $\nu>0$ such that $\int_{\nu}^{1 / \nu} \mu_{t}(s) \mathrm{d} s \geq \frac{\kappa(t)}{2}$ for every $t \in[a, b]$.

Remark 3.1. (i) The conditions $\left(M_{1}\right)-\left(M_{8}\right)$ are quoted from [8]. As is shown in [8], the following function

$$
\mu_{t}(s)=\frac{1}{[\varepsilon(t)]^{2}} e^{-\frac{s}{\varepsilon(t)}} \text { with } \varepsilon(t)=\frac{1}{4}\left[\frac{\pi}{2}-\arctan (t)\right]
$$

satisfies above mentioned conditions $\left(M_{1}\right)-\left(M_{8}\right)$.

(ii) Condition $\left(M_{2}\right)$ implies the continuous embedding: $\mathcal{M}_{\tau}^{\sigma} \hookrightarrow \mathcal{M}_{t}^{\sigma}$ for all $\sigma \in \mathbb{R}$ and $t>\tau$, with

$$
\|\eta\|_{\mathcal{M}_{t}^{\sigma}}^{2} \leq K_{\tau}(t)\|\eta\|_{\mathcal{M}_{\tau}^{\sigma}}^{2}, \forall \eta \in \mathcal{M}_{\tau}^{\sigma} .
$$

Therefore, $\mathcal{H}_{\tau}^{\sigma} \hookrightarrow \mathcal{H}_{t}^{\sigma}$ for all $\sigma \in \mathbb{R}$ and $t>\tau$.

Now, we quote some known results in recent literatures [7, 8], which are the bases of our arguments. Let us begin with the definition of weak solution.

Definition 3.2. [7] Let $T>\tau \in \mathbb{R}$, and $z_{\tau}=\left(u_{\tau}, v_{\tau}, \eta_{\tau}\right) \in \mathcal{H}_{\tau}$ be a fixed vector. A function

$$
z(t)=\left(u(t), \partial_{t} u(t), \eta^{t}\right) \in \mathcal{H}_{t} \text { for a.e. } t \in[\tau, T]
$$

is called a weak solution of problem (1.4)-(1.6) on interval $[\tau, T]$ if $u(\tau)=u_{\tau}, \partial_{t} u(\tau)=v_{\tau}$ and 
(i) $u \in L^{\infty}\left(\tau, T ; H^{1}\right), \partial_{t} u \in L^{\infty}(\tau, T ; H), \partial_{t t} u \in L^{1}\left(\tau, T ; H^{-1}\right)$;

(ii) the function $\eta^{t}$ fulfills the representation formula (1.5);

(iii) the function $u(t)$ fulfills (1.4) in the weak sense, i.e.,

$$
\left\langle\partial_{t t} u(t), \phi\right\rangle+\langle u(t), \phi\rangle_{1}+\int_{0}^{\infty} \mu_{t}(s)\left\langle\eta^{t}(s), \phi\right\rangle_{1} \mathrm{~d} s+\langle f(u(t)), \phi\rangle=\langle g, \phi\rangle
$$

for almost every $t \in[\tau, T]$ and every $\phi \in H^{1}$.

Theorem 3.3. [7] Let Assumption 3.1 be valid. Then for every $T>\tau \in \mathbb{R}$, and every $z_{\tau}=$ $\left(u_{\tau}, v_{\tau}, \eta_{\tau}\right) \in \mathcal{H}_{\tau}$, problem (1.4)-(1.6) admits a unique weak solution $z(t)=\left(u(t), \partial_{t} u(t), \eta^{t}\right)$ on $[\tau, T]$, with

$$
\left(u, \partial_{t} u\right) \in C\left([\tau, T] ; H^{1} \times H\right), \eta^{t} \in \mathcal{M}_{t}, \quad \forall t \in[\tau, T] .
$$

Moreover, for any two weak solutions $z_{1}(t)$ and $z_{2}(t)$ on $[\tau, T]$ with $\left\|z_{1}(\tau)\right\|_{\mathcal{H}_{\tau}}+\left\|z_{2}(\tau)\right\|_{\mathcal{H}_{\tau}} \leq R$,

$$
\left\|z_{1}(t)-z_{2}(t)\right\|_{\mathcal{H}_{t}} \leq \mathcal{Q}(R) e^{\mathcal{Q}(R)(t-\tau)}\left\|z_{1}(\tau)-z_{2}(\tau)\right\|_{\mathcal{H}_{\tau}}, \quad \forall t \in[\tau, T],
$$

where $\mathcal{Q}$ is an increasing positive function independent of $t$.

3.2. Main results. Under Assumption 3.1, we define the mapping

$$
U(t, \tau): \mathcal{H}_{\tau} \rightarrow \mathcal{H}_{t}, \quad U(t, \tau) z_{\tau}=z(t), \forall z_{\tau} \in \mathcal{H}_{\tau}, t \geq \tau,
$$

where $z(t)$ is the weak solution of problem (1.4)-(1.6) corresponding to the initial data $z_{\tau} \in \mathcal{H}_{\tau}$. By Theorem 3.3, the two-parameter family $\{U(t, \tau) \mid t \geq \tau\}$ constitutes a process acting on time-dependent Banach spaces $\left\{\mathcal{H}_{t}\right\}_{t \in \mathbb{R}}$.

Lemma 3.4. [8] Let Assumption 3.1 be valid. Then the process $U(t, \tau): \mathcal{H}_{\tau} \rightarrow \mathcal{H}_{t}$ generated by problem (1.4)-(1.6) possesses the invariant time-dependent global attractor $\mathcal{A}=\{A(t)\}_{t \in \mathbb{R}}$, with $A(t) \subset \mathcal{H}_{t}^{1}$ for each $t \in \mathbb{R}$, and

$$
\sup _{t \in \mathbb{R}}\|A(t)\|_{\mathcal{H}_{t}^{1}}<\infty
$$

Now, we state the main results of the present paper, and its proof will be given in Section 5 after some delicate technique preparations in Section 4.

Theorem 3.5. Let Assumption 3.1 be valid. Then the process $U(t, \tau): \mathcal{H}_{\tau} \rightarrow \mathcal{H}_{t}$ generated by problem (1.4)-(1.6) admits a time-dependent exponential attractor $\mathcal{E}=\{E(t)\}_{t \in \mathbb{R}}$, with $E(t) \subset \mathcal{H}_{t}^{1}$ for each $t \in \mathbb{R}$, and

$$
\sup _{t \in \mathbb{R}}\|E(t)\|_{\mathcal{H}_{t}^{1}}<\infty .
$$

Corollary 3.6. Let Assumption 3.1 be valid. Then the fractal dimension of the invariant time-dependent global attractor $\mathcal{A}=\{A(t)\}_{t \in \mathbb{R}}$ given by Lemma 3.4 is uniformly bounded, that is,

$$
\sup _{t \in \mathbb{R}} \operatorname{dim}_{f}\left(A(t) ; \mathcal{H}_{t}\right) \leq \sup _{t \in \mathbb{R}} \operatorname{dim}_{f}\left(E(t) ; \mathcal{H}_{t}\right)<+\infty .
$$


EXPONENTIAL ATTRACTOR FOR THE VISCOELASTIC WAVE MODEL WITH TIME-DEPENDENT MEMORY KERNELS ${ }^{7} 15$

\section{Some Key estimates}

We first quote some known results (Lemmas 4.1 to 4.6) coming from literature [7, 8], which will be the stating point of our argument.

Lemma 4.1. [8] (Gronwall-type lemma in integral form) Let $\tau \in \mathbb{R}$ be fixed, and $\Lambda:[\tau, \infty) \rightarrow \mathbb{R}$ be a continuous function. Assume that, for some $\epsilon>0$ and every $b>a \geq \tau$,

$$
\Lambda(b)+2 \epsilon \int_{a}^{b} \Lambda(y) \mathrm{d} y \leq \Lambda(a)+\int_{a}^{b} q_{1}(y) \Lambda(y) \mathrm{d} y+\int_{a}^{b} q_{2}(y) \mathrm{d} y
$$

where $q_{1}, q_{2}$ are locally summable nonnegative functions on $[\tau, \infty)$ satisfying

$$
\int_{a}^{b} q_{1}(y) \mathrm{d} y \leq \epsilon(b-a)+c_{1} \text { and } \sup _{t \geq \tau} \int_{t}^{t+1} q_{2}(y) \mathrm{d} y \leq c_{2}
$$

for some $c_{1}, c_{2} \geq 0$. Then, we have

$$
\Lambda(t) \leq e^{c_{1}}\left[|\Lambda(\tau)| e^{-\epsilon(t-\tau)}+\frac{c_{2} e^{\epsilon}}{1-e^{-\epsilon}}\right], \forall t \geq \tau
$$

We consider the following problem

$$
\begin{aligned}
& \partial_{t t} p(t)+A p(t)+\int_{0}^{\infty} \mu_{t}(s) A \psi^{t}(s) \mathrm{d} s+\gamma(t)=0, \quad t>\tau, \\
& \psi^{t}(s)= \begin{cases}p(t)-p(t-s), & s \leq t-\tau, \\
\psi_{\tau}(s-t+\tau)+p(t)-p_{\tau}, & s>t-\tau,\end{cases} \\
& p(\tau)=p_{\tau}, \quad \partial_{t} p(\tau)=q_{\tau}, \psi^{\tau}=\psi_{\tau},
\end{aligned}
$$

where $\gamma$ is a certain forcing term (possibly depending on $p$ ) and $\left(p_{\tau}, q_{\tau}, \psi_{\tau}\right) \in \mathcal{H}_{\tau}$. Assuming that problem (4.1)-(4.3) admits a global solution $\left(p(t), \partial_{t} p(t), \psi^{t}\right) \in \mathcal{H}_{t}$ for all $t \in[\tau, \infty)$.

Lemma 4.2. [7] Let Assumption 3.1: (ii) be valid. For any fixed $\sigma \in \mathbb{R}$ and every $T>\tau \in \mathbb{R}$, if also

$$
p \in W^{1, \infty}\left(\tau, T ; H^{\sigma+1}\right) \text { and } \psi_{\tau} \in \mathcal{M}_{\tau}^{\sigma}
$$

then for all $\tau \leq a \leq b \leq T$,

$$
\left\|\psi^{b}\right\|_{\mathcal{M}_{b}^{\sigma}}^{2}-\int_{a}^{b} \int_{0}^{\infty}\left[\partial_{t} \mu_{t}(s)+\partial_{s} \mu_{t}(s)\right]\left\|\psi^{t}(s)\right\|_{\sigma+1}^{2} \mathrm{~d} s \mathrm{~d} t \leq\left\|\psi^{a}\right\|_{\mathcal{M}_{a}^{\sigma}}^{2}+2 \int_{a}^{b}\left\langle\partial_{t} p(t), \psi^{t}\right\rangle_{\mathcal{M}_{t}^{\sigma}} \mathrm{d} t .
$$

Lemma 4.3. [8] Let Assumption 3.1: (ii) be valid, and the global solution $\left(p(t), \partial_{t} p(t), \psi^{t}\right)$ of problem (4.1)-(4.3) be sufficiently regular and let the functionals

$$
\begin{aligned}
& \Phi(t)=2\left\langle p(t), \partial_{t} p(t)\right\rangle \\
& \Psi(t)=-\frac{2}{\kappa(t)} \int_{0}^{\infty} \mu_{t}(s)\left\langle\psi^{t}(s), \partial_{t} p(t)\right\rangle \mathrm{d} s .
\end{aligned}
$$


Then, for every $\varpi \in(0,1]$ and every $b>a \geq \tau$, we have

$$
\begin{aligned}
\Phi(b)+(2-\varpi) \int_{a}^{b}\|p(t)\|_{1}^{2} \mathrm{~d} t \leq & \Phi(a)+2 \int_{a}^{b}\left\|\partial_{t} p(t)\right\|^{2} \mathrm{~d} t \\
& +\frac{1}{\varpi} \int_{a}^{b} \kappa(t)\left\|\psi^{t}\right\|_{\mathcal{M}_{t}}^{2} \mathrm{~d} t-2 \int_{a}^{b}\langle\gamma(t), p(t)\rangle \mathrm{d} t,
\end{aligned}
$$

and

$$
\begin{aligned}
\Psi(b)+\int_{a}^{b}\left\|\partial_{t} p(t)\right\|^{2} \mathrm{~d} t \leq & \Psi(a)-M \int_{a}^{b} \int_{0}^{\infty}\left[\partial_{t} \mu_{t}(s)+\partial_{s} \mu_{t}(s)\right]\left\|\psi^{t}(s)\right\|_{1}^{2} \mathrm{~d} s \mathrm{~d} t \\
& +\varpi \int_{a}^{b}\|p(t)\|_{1}^{2} \mathrm{~d} t+\frac{C}{\varpi} \int_{a}^{b} \kappa(t)\left\|\psi^{t}\right\|_{\mathcal{M}_{t}}^{2} \mathrm{~d} t \\
& +\int_{a}^{b} \frac{2}{\kappa(t)} \int_{0}^{\infty} \mu_{t}(s)\left\langle\psi^{t}(s), \gamma(t)\right\rangle \mathrm{d} s \mathrm{~d} t,
\end{aligned}
$$

where $M$ and $C$ are positive constants depending only on the structural assumptions on the memory kernel.

Remark 4.4. (i) In the following in our arguments, Lemma 4.3 is always used to the Galerkin approximations which are of enough regularity.

(ii) By conditions $\left(M_{1}\right),\left(M_{4}\right)-\left(M_{5}\right)$ one easily sees that

$$
|\Phi(t)|+|\Psi(t)| \leq C\left\|\left(p(t), \partial_{t} p(t), \psi^{t}\right)\right\|_{\mathcal{H}_{t}}^{2}, \quad \forall t \geq \tau .
$$

Lemma 4.5. [8] Let Assumption 3.1 be valid, and $z_{\tau} \in \mathcal{H}_{\tau}$ with $\left\|z_{\tau}\right\|_{\mathcal{H}_{\tau}} \leq R$. Then there exist positive constants $\omega$ and $R_{0}$, which are independent of $R$, such that

$$
\mathcal{E}(t, \tau):=\frac{1}{2}\left\|U(t, \tau) z_{\tau}\right\|_{\mathcal{H}_{t}}^{2} \leq \mathcal{Q}(R) e^{-\omega(t-\tau)}+R_{0}, \quad \forall t \geq \tau .
$$

That is, the family $\left\{\mathbb{B}_{t}\left(R_{1}\right)\right\}_{t \in \mathbb{R}}$, with $R_{1}>\sqrt{2 R_{0}}$ is a uniformly time-dependent absorbing set of the process $U(t, \tau)$.

Following the standard method given in [12], we split the nonlinearity $f$ into the sum

$$
f(u)=f_{0}(u)+f_{1}(u)
$$

where $f_{1} \in C^{2}(\mathbb{R})$ is globally Lipschitz with $f_{1}(0)=0$, while $f_{0} \in C^{2}(\mathbb{R})$ vanishes inside $[-1,1]$ and

$$
\left|f_{0}^{\prime \prime}(u)\right| \leq C|u|, \quad f_{0}^{\prime}(u) \geq 0
$$

for some positive constant $C$. Then we decompose the solution $U(t, \tau) z_{\tau}$ as the sum

$$
U(t, \tau) z_{\tau}=U_{0}(t, \tau) z_{\tau}+U_{1}(t, \tau) z_{\tau},
$$

where $U_{0}(t, \tau) z_{\tau}=\left(v(t), \partial_{t} v(t), \xi^{t}\right)$ solves the problem

$$
\left\{\begin{array}{l}
\partial_{t t} v(t)+A v(t)+\int_{0}^{\infty} \mu_{t}(s) A \xi^{t}(s) \mathrm{d} s+f_{0}(v(t))=0 \\
U_{0}(\tau, \tau) z_{\tau}=z_{\tau}
\end{array}\right.
$$


EXPONENTIAL ATTRACTOR FOR THE VISCOELASTIC WAVE MODEL WITH TIME-DEPENDENT MEMORY KERNELS ${ }_{17}$

where

$$
\xi^{t}(s)= \begin{cases}v(t)-v(t-s), & s \leq t-\tau, \\ \xi_{\tau}(s-t+\tau)+v(t)-v_{\tau}, & s>t-\tau,\end{cases}
$$

and $U_{1}(t, \tau) z_{\tau}=\left(w(t), \partial_{t} w(t), \zeta^{t}\right)$ solves the problem

$$
\left\{\begin{array}{l}
\partial_{t t} w(t)+A w(t)+\int_{0}^{\infty} \mu_{t}(s) A \zeta^{t}(s) \mathrm{d} s+f_{0}(u(t))-f_{0}(v(t))+f_{1}(u(t))=g, \\
U_{1}(\tau, \tau) z_{\tau}=0
\end{array}\right.
$$

where

$$
\zeta^{t}(s)= \begin{cases}w(t)-w(t-s), & s \leq t-\tau \\ \zeta_{\tau}(s-t+\tau)+w(t)-w_{\tau}, & s>t-\tau\end{cases}
$$

Lemma 4.6. [8] Let Assumption 3.1 be valid, and $z_{\tau} \in \mathcal{H}_{\tau}$ with $\left\|z_{\tau}\right\|_{\mathcal{H}_{\tau}} \leq R$. Then for every $t \geq \tau$,

$$
\begin{aligned}
& \left\|U_{0}(t, \tau) z_{\tau}\right\|_{\mathcal{H}_{t}}^{2} \leq \mathcal{Q}(R) e^{-\omega(t-\tau)}, \\
& \left\|U_{1}(t, \tau) z_{\tau}\right\|_{\mathcal{H}_{t}^{1 / 3}}^{2} \leq \mathcal{Q}(R),
\end{aligned}
$$

hereafter $\omega>0$ is as shown in Lemma 4.5 .

Now, based on Lemmas 4.1 to 4.6, we further establish some new estimates which will play key roles for our applying Theorem 2.5 and its corollary to problem (1.4)-(1.6) to establish the existence of the time-dependent exponential attractors.

Lemma 4.7. Let Assumption 3.1 be valid, and $z_{\tau} \in \mathcal{H}_{\tau}^{1 / 3}$ with $\left\|z_{\tau}\right\|_{\mathcal{H}_{\tau}} \leq R$. Then for every $t \geq \tau$, we have

$$
\left\|U(t, \tau) z_{\tau}\right\|_{\mathcal{H}_{t}^{1 / 3}}^{2} \leq \mathcal{Q}\left(R+\left\|z_{\tau}\right\|_{\mathcal{H}_{\tau}^{1 / 3}}\right) e^{-\omega(t-\tau)}+\mathcal{Q}(R) .
$$

Proof. For any $t \geq \tau$, we define the functionals of the solutions $\left(u(t), \partial_{t} u(t), \eta^{t}\right)=U(t, \tau) z_{\tau}$ as follows:

$$
\begin{aligned}
& \mathcal{E}_{1 / 3}(t, \tau)=\frac{1}{2}\left\|U(t, \tau) z_{\tau}\right\|_{\mathcal{H}_{t}^{1 / 3}}^{2}, \\
& \mathcal{L}_{1 / 3}(t)=L_{1 / 3}(t)+\left\|\eta^{t}\right\|_{\mathcal{M}_{t}^{1 / 3}}^{2}, \\
& \Lambda_{1 / 3}(t)=\mathcal{L}_{1 / 3}(t)+2 \epsilon[\Phi(t)+4 \Psi(t)],
\end{aligned}
$$

where $\epsilon \in(0,1], \Phi$ and $\Psi$ are as shown in (4.4)-(4.5), with $\left(p(t), \partial_{t} p(t), \psi^{t}\right)=\left(A^{1 / 6} u(t), \partial_{t} A^{1 / 6} u(t), A^{1 / 6} \eta^{t}\right)$, and the functional

$$
L_{1 / 3}(t)=\|u(t)\|_{4 / 3}^{2}+\left\|\partial_{t} u(t)\right\|_{1 / 3}^{2}+2\left\langle\gamma(t), A^{1 / 6} u(t)\right\rangle, \text { with } \gamma(t)=A^{1 / 6}(f(u(t))-g) .
$$

By condition (3.1) and Lemma 4.5, we have

$$
\|f(u)\| \leq C\left\|1+|u|^{3}\right\| \leq C\left(1+\|u\|_{L^{6}}^{3}\right) \leq C\left(1+\|u\|_{1}^{3}\right) \leq \mathcal{Q}(R),
$$

which implies that

$$
2 \mid\left\langle\gamma(t), A^{1 / 6} u(t) \mid\right\rangle \leq 2[\|f(u)\|+\|g\|]\|u\|_{2 / 3} \leq \frac{1}{4}\|u(t)\|_{4 / 3}^{2}+\mathcal{Q}(R),
$$


and hence,

$$
\frac{3}{2} \mathcal{E}_{1 / 3}(t, \tau)-\mathcal{Q}(R) \leq \mathcal{L}_{1 / 3}(t) \leq \frac{5}{2} \mathcal{E}_{1 / 3}(t, \tau)+\mathcal{Q}(R)
$$

By formula (4.8),

$$
|\Phi(t)|+|\Psi(t)| \leq C \mathcal{E}_{1 / 3}(t, \tau), \quad \forall t \geq \tau .
$$

The combination of (4.16) and (4.18)-(4.19) yields

$$
\mathcal{E}_{1 / 3}(t, \tau)-\mathcal{Q}(R) \leq \Lambda_{1 / 3}(t) \leq 3 \mathcal{E}_{1 / 3}(t, \tau)+\mathcal{Q}(R)
$$

for $\epsilon>0$ suitably small. Taking the multiplier $2 A^{1 / 3} \partial_{t} u$ in Eq. (1.4) gives

$$
\frac{\mathrm{d}}{\mathrm{d} t} L_{1 / 3}(t)+2\left\langle\eta^{t}, \partial_{t} u(t)\right\rangle_{\mathcal{M}_{t}^{1 / 3}}=2\left\langle f^{\prime}(u(t)) \partial_{t} u(t), A^{1 / 3} u(t)\right\rangle:=I_{1}(t)+I_{2}(t)+I_{3}(t),
$$

where

$$
\begin{aligned}
& I_{1}=2\left\langle\left[f_{0}^{\prime}(u)-f_{0}^{\prime}(v)\right] \partial_{t} u, A^{1 / 3} u\right\rangle \\
& I_{2}=2\left\langle f_{0}^{\prime}(v) \partial_{t} u, A^{1 / 3} u\right\rangle \\
& I_{3}=2\left\langle f_{1}^{\prime}(u) \partial_{t} u, A^{1 / 3} u\right\rangle
\end{aligned}
$$

By Lemma 4.5, estimates (4.10), (4.13)-(4.14) and the Sobolev embedding

$$
H^{1} \hookrightarrow L^{6}, H^{4 / 3} \hookrightarrow L^{18}, H^{2 / 3} \hookrightarrow L^{18 / 5}, H^{1 / 3} \hookrightarrow L^{18 / 7},
$$

we have

$$
\begin{aligned}
\left|I_{1}\right| & \leq C \int_{\Omega}(|u|+|v|)|w|\left\|\partial_{t} u\right\| A^{1 / 3} u \mid \mathrm{d} x \\
& \leq C\left(\|u\|_{L^{6}}+\|v\|_{L^{6}}\right)\|w\|_{L^{18}}\left\|\partial_{t} u\right\|\left\|A^{1 / 3} u\right\|_{L^{18 / 5}} \\
& \leq \alpha\|u\|_{4 / 3}^{2}+\frac{\mathcal{Q}(R)}{\alpha}, \forall \alpha \in(0,1], \\
\left|I_{2}\right| & \leq C \int_{\Omega}|v|^{2}\left|\partial_{t} u \| A^{1 / 3} u\right| \mathrm{d} x \\
& \leq C\|v\|_{L^{6}}^{2}\left\|\partial_{t} u\right\|_{L^{18 / 7}}\left\|A^{1 / 3} u\right\|_{L^{18 / 5}} \\
& \leq C\|v\|_{1}^{2}\left[\left\|\partial_{t} u\right\|_{1 / 3}^{2}+\|u\|_{4 / 3}^{2}\right],
\end{aligned}
$$

and

$$
\left|I_{3}\right| \leq C\left\|\partial_{t} u\right\|\left\|A^{1 / 3} u\right\| \leq \mathcal{Q}(R)\|u\|_{4 / 3} \leq \alpha\|u\|_{4 / 3}^{2}+\frac{\mathcal{Q}(R)}{\alpha}, \quad \forall \alpha \in(0,1] .
$$

Inserting above estimates into (4.21) and making use of (4.18) receive

$$
\frac{\mathrm{d}}{\mathrm{d} t} L_{1 / 3}(t)+2\left\langle\eta^{t}, \partial_{t} u\right\rangle_{\mathcal{M}_{t}^{1 / 3}} \leq q_{1}(t) \mathcal{L}_{1 / 3}(t)+\mathcal{Q}(R) q_{1}(t)+\frac{\mathcal{Q}(R)}{\alpha}, \quad \forall \alpha \in(0,1],
$$


EXPONENTIAL ATTRACTOR FOR THE VISCOELASTIC WAVE MODEL WITH TIME-DEPENDENT MEMORY KERNELS ${ }^{9} 19$ where $q_{1}(t)=C\left(\alpha+\|v(t)\|_{1}^{2}\right)$. Integrating inequality (4.23) over $[a, b]$, with $b \geq a \geq \tau$, yields

$$
\begin{aligned}
& L_{1 / 3}(b)+2 \int_{a}^{b}\left\langle\eta^{t}, \partial_{t} u(t)\right\rangle_{\mathcal{M}_{t}^{1 / 3}} \mathrm{~d} t \\
\leq & L_{1 / 3}(a)+\int_{a}^{b} q_{1}(t) \mathcal{L}_{1 / 3}(t) \mathrm{d} t+\int_{a}^{b}\left(q_{1}(t)+\frac{\mathcal{Q}(R)}{\alpha}\right) \mathrm{d} t, \quad \forall \alpha \in(0,1] .
\end{aligned}
$$

It follows form Lemma 4.2 (taking $\sigma=1 / 3$ there) that

$$
\begin{aligned}
& \left\|\eta^{b}\right\|_{\mathcal{M}_{b}^{1 / 3}}^{2}-\int_{a}^{b} \int_{0}^{\infty}\left[\partial_{t} \mu_{t}(s)+\partial_{s} \mu_{t}(s)\right]\left\|\eta^{t}(s)\right\|_{4 / 3}^{2} \mathrm{~d} s \mathrm{~d} t \\
\leq & \left\|\eta^{a}\right\|_{\mathcal{M}_{a}^{1 / 3}}^{2}+2 \int_{a}^{b}\left\langle\partial_{t} u(t), \eta^{t}\right\rangle_{\mathcal{M}_{t}^{1 / 3}} \mathrm{~d} t .
\end{aligned}
$$

Adding above two inequalities together we obtain

$$
\begin{aligned}
& \mathcal{L}_{1 / 3}(b)-\int_{a}^{b} \int_{0}^{\infty}\left[\partial_{t} \mu_{t}(s)+\partial_{s} \mu_{t}(s)\right]\left\|\eta^{t}(s)\right\|_{4 / 3}^{2} \mathrm{~d} s \mathrm{~d} t \\
\leq & \mathcal{L}_{1 / 3}(a)+\int_{a}^{b} q_{1}(t) \mathcal{L}_{1 / 3}(t) \mathrm{d} t+\int_{a}^{b}\left(q_{1}(t)+\frac{\mathcal{Q}(R)}{\alpha}\right) \mathrm{d} t, \quad \forall \alpha \in(0,1] .
\end{aligned}
$$

Exploiting estimates (4.6)-(4.7) ( taking $\varpi=\frac{1}{20}$ there), we get

$$
\begin{aligned}
& \Phi(b)+4 \Psi(b)+\frac{7}{4} \int_{a}^{b}\|u(t)\|_{4 / 3}^{2} \mathrm{~d} t+2 \int_{a}^{b}\left\|\partial_{t} u(t)\right\|_{1 / 3}^{2} \mathrm{~d} t \\
\leq & \Phi(a)+4 \Psi(a)-4 M \int_{a}^{b} \int_{0}^{\infty}\left[\partial_{t} \mu_{t}(s)+\partial_{s} \mu_{t}(s)\right]\left\|\eta^{t}(s)\right\|_{4 / 3}^{2} \mathrm{~d} s \mathrm{~d} t \\
& +C \int_{a}^{b} \kappa(t)\left\|\eta^{t}\right\|_{\mathcal{M}_{t}^{1 / 3}}^{2} \mathrm{~d} t-2 \int_{a}^{b}\left\langle\gamma(t), A^{1 / 6} u(t)\right\rangle \mathrm{d} t \\
& +8 \int_{a}^{b} \frac{1}{\kappa(t)} \int_{0}^{\infty} \mu_{t}(s)\left\langle A^{1 / 6} \eta^{t}(s), \gamma(t)\right\rangle \mathrm{d} s \mathrm{~d} t
\end{aligned}
$$

Due to

$$
\|\gamma(t)\|_{-1}=\left\|A^{-1 / 3}(f(u)-g)\right\| \leq C(\|f(u(t))\|+\|g\|) \leq \mathcal{Q}(R),
$$

we have

$$
\begin{aligned}
-2 \int_{a}^{b}\left\langle\gamma(t), A^{1 / 6} u(t)\right\rangle \mathrm{d} t & \leq 2 \int_{a}^{b}\|\gamma(t)\|_{-1}\|u(t)\|_{4 / 3} \mathrm{~d} t \\
& \leq \frac{1}{4} \int_{a}^{b}\|u(t)\|_{4 / 3}^{2} \mathrm{~d} t+\mathcal{Q}(R)(b-a),
\end{aligned}
$$


and by conditions $\left(M_{1}\right)$ and $\left(M_{5}\right)$, we have

$$
\begin{aligned}
& 8 \int_{a}^{b} \frac{1}{\kappa(t)} \int_{0}^{\infty} \mu_{t}(s)\left\langle A^{1 / 6} \eta^{t}(s), \gamma(t)\right\rangle \mathrm{d} s \mathrm{~d} t \\
\leq & 8 \int_{a}^{b} \frac{1}{\kappa(t)}\|\gamma(t)\|_{-1}\left(\int_{0}^{\infty} \mu_{t}(s)\left\|\eta^{t}(s)\right\|_{4 / 3} \mathrm{~d} s\right) \mathrm{d} t \\
\leq & \mathcal{Q}(R) \int_{a}^{b} \frac{1}{\kappa(t)} \sqrt{\kappa(t)}\left\|\eta^{t}\right\|_{\mathcal{M}_{t}^{1 / 3}} \mathrm{~d} t \\
\leq & \mathcal{Q}(R)(b-a)+\mathcal{Q}(R) \int_{a}^{b} \kappa(t)\left\|\eta^{t}\right\|_{\mathcal{M}_{t}^{1 / 3}}^{2} \mathrm{~d} t .
\end{aligned}
$$

Inserting estimates (4.26)-(4.27) into (4.25) and making use of condition $\left(M_{5}\right)$ and (4.20) turn out

$$
\begin{aligned}
& \Phi(b)+4 \Psi(b)+\int_{a}^{b} \Lambda_{1 / 3}(t) \mathrm{d} t \\
\leq & \Phi(a)+4 \Psi(a)-4 M \int_{a}^{b} \int_{0}^{\infty}\left[\partial_{t} \mu_{t}(s)+\partial_{s} \mu_{t}(s)\right]\left\|\eta^{t}(s)\right\|_{4 / 3}^{2} \mathrm{~d} s \mathrm{~d} t \\
& +\mathcal{Q}(R) \int_{a}^{b} \kappa(t)\left\|\eta^{t}\right\|_{\mathcal{M}_{t}^{1 / 3}}^{2} \mathrm{~d} t+\mathcal{Q}(R)(b-a) .
\end{aligned}
$$

The combination of (4.24) and (4.28) yields

$$
\begin{aligned}
& \Lambda_{1 / 3}(b)+2 \epsilon \int_{a}^{b} \Lambda_{1 / 3}(t) \mathrm{d} t+\mathcal{J} \\
\leq & \Lambda_{1 / 3}(a)+\int_{a}^{b} q_{1}(t) \mathcal{L}_{1 / 3}(t) \mathrm{d} t+\mathcal{Q}(R) \int_{a}^{b}\left(q_{1}(t)+\frac{1}{\alpha}\right) \mathrm{d} t \\
\leq & \Lambda_{1 / 3}(a)+\frac{5}{2} \int_{a}^{b} q_{1}(t) \Lambda_{1 / 3}(t) \mathrm{d} t+\mathcal{Q}(R) \int_{a}^{b}\left(q_{1}(t)+\frac{1}{\alpha}\right) \mathrm{d} t,
\end{aligned}
$$

where

$$
\begin{aligned}
\mathcal{J} & =-(1-8 \epsilon M) \int_{a}^{b} \int_{0}^{\infty}\left[\partial_{t} \mu_{t}(s)+\partial_{s} \mu_{t}(s)\right]\left\|\eta^{t}(s)\right\|_{4 / 3}^{2} \mathrm{~d} s \mathrm{~d} t-2 \epsilon \mathcal{Q}(R) \int_{a}^{b} \kappa(t)\left\|\eta^{t}\right\|_{\mathcal{M}_{t}^{1 / 3}}^{2} \mathrm{~d} t \\
& \geq(\delta(1-8 \epsilon M)-2 \epsilon \mathcal{Q}(R)) \int_{a}^{b} \kappa(t)\left\|\eta^{t}\right\|_{\mathcal{M}_{t}^{1 / 3}}^{2} \mathrm{~d} t \geq 0
\end{aligned}
$$

for $\epsilon \in(0,1]$ suitably small. Taking $\alpha: C \alpha=\omega \leq \epsilon$, a simple calculation shows that

$$
\begin{aligned}
& \frac{5}{2} \int_{a}^{b} q_{1}(t) \mathrm{d} t \leq C \alpha(b-a)+\mathcal{Q}(R) \int_{a}^{b} e^{-\omega(t-\tau)} d t \leq \omega(b-a)+\mathcal{Q}(R), \\
& \sup _{t \geq \tau} \int_{t}^{t+1} Q(R)\left(q_{1}(s)+\frac{1}{\alpha}\right) \mathrm{d} s \leq \mathcal{Q}(R)\left(\omega+\frac{Q(R)}{\omega}\right)=Q(R) .
\end{aligned}
$$

Therefore, applying Lemma 4.1 to (4.29) and making use of (4.20) give the conclusion of Lemma 4.7.

For any fixed $\tau \in \mathbb{R}$ and $z_{\tau} \in \mathcal{H}_{\tau}^{1 / 3}$ with $\left\|z_{\tau}\right\|_{\mathcal{H}_{\tau}^{1 / 3}} \leq R$, we still write

$$
U(t, \tau) z_{\tau}=U_{0}(t, \tau) z_{\tau}+U_{1}(t, \tau) z_{\tau},
$$


EXPONENTIAL ATTRACTOR FOR THE VISCOELASTIC WAVE MODEL WITH TIME-DEPENDENT MEMORY KERNELS ${ }^{1}{ }_{2} 1$ where $U_{0}(t, \tau)$ and $U_{1}(t, \tau)$ are the solution operators of problems (4.11) and (4.12), respectively, with

$$
f_{0}(u)=0 \text { and } f_{1}(u)=f(u) .
$$

It follows from Lemma 4.7 that, for every $z_{\tau} \in \mathcal{H}_{\tau}^{1 / 3}$ with $\left\|z_{\tau}\right\|_{\mathcal{H}_{\tau}^{1 / 3}} \leq R$,

$$
\left\|U(t, \tau) z_{\tau}\right\|_{\mathcal{H}_{t}^{1 / 3}}^{2} \leq \mathcal{Q}(R), \forall t \geq \tau .
$$

Thus, by using the same argument as Lemma 8.1 in [8], we have (the proof is omitted here)

Lemma 4.8. Let Assumption 3.1 be valid, and $z_{\tau} \in \mathcal{H}_{\tau}^{1 / 3}$ with $\left\|z_{\tau}\right\|_{\mathcal{H}_{\tau}^{1 / 3}} \leq R$. Then for every $t \geq \tau$,

$$
\begin{aligned}
\left\|U_{0}(t, \tau) z_{\tau}\right\|_{\mathcal{H}_{t}}^{2} & \leq \mathcal{Q}(R) e^{-\omega(t-\tau)}, \\
\left\|U_{1}(t, \tau) z_{\tau}\right\|_{\mathcal{H}_{t}^{1}}^{2} & \leq \mathcal{Q}(R) .
\end{aligned}
$$

Based on Lemma 4.8, we give a further delicate estimate.

Lemma 4.9. Let Assumption 3.1 be valid, and $z_{\tau} \in \mathcal{H}_{\tau}^{1}$ with $\left\|z_{\tau}\right\|_{\mathcal{H}_{\tau}^{1 / 3}} \leq R$. Then

$$
\left\|U(t, \tau) z_{\tau}\right\|_{\mathcal{H}_{t}^{1}}^{2} \leq \mathcal{Q}\left(R+\left\|z_{\tau}\right\|_{\mathcal{H}_{\tau}^{1}}\right) e^{-\omega(t-\tau)}+\mathcal{Q}(R), \quad \forall t \geq \tau .
$$

Proof. For any $t \geq \tau$, we define the functionals of the solutions $\left(u(t), \partial_{t} u(t), \eta^{t}\right)=U(t, \tau) z_{\tau}$ as follows:

$$
\begin{aligned}
& \mathcal{E}_{1}(t, \tau)=\frac{1}{2}\left\|U(t, \tau) z_{\tau}\right\|_{\mathcal{H}_{t}^{1}}^{2}, \\
& \mathcal{L}_{1}(t)=L_{1}(t)+\left\|\eta^{t}\right\|_{\mathcal{M}_{t}^{1}}^{2}, \\
& \Lambda_{1}(t)=\mathcal{L}_{1}(t)+2 \epsilon[\Phi(t)+4 \Psi(t)],
\end{aligned}
$$

where $\epsilon \in(0,1], \Phi$ and $\Psi$ are defined as in (4.4)-(4.5), with $\left(p(t), \partial_{t} p(t), \psi^{t}\right)=\left(A^{1 / 2} u(t), \partial_{t} A^{1 / 2} u(t), A^{1 / 2} \eta^{t}\right)$, and the functional

$$
L_{1}(t)=\|u(t)\|_{2}^{2}+\left\|\partial_{t} u(t)\right\|_{1}^{2}+2\left\langle\gamma(t), A^{1 / 2} u\right\rangle, \text { with } \gamma(t)=A^{1 / 2}(f(u)-g) .
$$

By (4.17),

$$
2\left|\left\langle\gamma(t), A^{1 / 2} u\right\rangle\right| \leq 2\|\gamma(t)\|_{-1}\left\|A^{1 / 2} u\right\|_{1} \leq C[\|f(u)\|+\|g\|]\|u\|_{2} \leq \frac{1}{4}\|u(t)\|_{2}^{2}+\mathcal{Q}(R) .
$$

Consequently,

$$
\frac{3}{2} \mathcal{E}_{1}(t, \tau)-\mathcal{Q}(R) \leq \mathcal{L}_{1}(t) \leq \frac{5}{2} \mathcal{E}_{1}(t, \tau)+\mathcal{Q}(R) .
$$

It follows from (4.8) that

$$
|\Phi(t)|+|\Psi(t)| \leq C \mathcal{E}_{1}(t, \tau), \quad \forall t \geq \tau,
$$

which combining with (4.35)-(4.36) gives

$$
\mathcal{E}_{1}(t, \tau)-\mathcal{Q}(R) \leq \Lambda_{1}(t) \leq 3 \mathcal{E}_{1}(t, \tau)+\mathcal{Q}(R)
$$


for $\epsilon \in(0,1]$ suitably small. Taking the multiplier $2 A \partial_{t} u$ in Eq. (1.4) gives

$$
\begin{aligned}
\frac{\mathrm{d}}{\mathrm{d} t} L_{1}(t)+2\left\langle\eta^{t}, \partial_{t} u(t)\right\rangle_{\mathcal{M}_{t}^{1}} & =2\left\langle f^{\prime}(u(t)) \partial_{t} u(t), A u(t)\right\rangle \\
& \leq C\left(1+\|u(t)\|_{L^{18}}^{2}\right)\left\|\partial_{t} u(t)\right\|_{L^{18 / 7}}\|A u(t)\| \\
& \leq \mathcal{Q}(R)\|u(t)\|_{2} \leq \alpha\|u(t)\|_{2}^{2}+\frac{\mathcal{Q}(R)}{\alpha}, \forall \alpha \in(0,1], t \geq \tau,
\end{aligned}
$$

where we have used condition (3.1), Sobolev embedding (4.22) and formula (4.31). Integrating above inequality over $[a, b]$, with $b \geq a \geq \tau$, gives

$$
L_{1}(b)+2 \int_{a}^{b}\left\langle\eta^{t}, \partial_{t} u(t)\right\rangle_{\mathcal{M}_{t}^{1}} \mathrm{~d} t \leq L_{1}(a)+\alpha \int_{a}^{b}\|u(t)\|_{2}^{2} \mathrm{~d} t+\frac{\mathcal{Q}(R)}{\alpha}(b-a), \quad \forall \alpha \in(0,1] .
$$

Applying Lemma 4.2 (taking $\sigma=1$ there) yields

$$
\left\|\eta^{b}\right\|_{\mathcal{M}_{b}^{1}}^{2}-\int_{a}^{b} \int_{0}^{\infty}\left[\partial_{t} \mu_{t}(s)+\partial_{s} \mu_{t}(s)\right]\left\|\eta^{t}(s)\right\|_{2}^{2} \mathrm{~d} s \mathrm{~d} t \leq\left\|\eta^{a}\right\|_{\mathcal{M}_{a}^{1}}^{2}+2 \int_{a}^{b}\left\langle\partial_{t} u(t), \eta^{t}\right\rangle_{\mathcal{M}_{t}^{1}} \mathrm{~d} t .
$$

Adding above two inequalities together turns out

$$
\begin{aligned}
& \mathcal{L}_{1}(b)-\int_{a}^{b} \int_{0}^{\infty}\left[\partial_{t} \mu_{t}(s)+\partial_{s} \mu_{t}(s)\right]\left\|\eta^{t}(s)\right\|_{2}^{2} \mathrm{~d} s \mathrm{~d} t \\
\leq & \mathcal{L}_{1}(a)+\alpha \int_{a}^{b}\|u(t)\|_{2}^{2} \mathrm{~d} t+\frac{\mathcal{Q}(R)}{\alpha}(b-a), \quad \forall \alpha \in(0,1] .
\end{aligned}
$$

Now, we give the estimates of the last term in the right hand side of the corresponding formulas (4.6)-(4.7), respectively. By (4.17),

$$
-2 \int_{a}^{b}\left\langle\gamma(t), A^{1 / 2} u(t)\right\rangle \mathrm{d} t \leq \frac{1}{20} \int_{a}^{b}\|u(t)\|_{2}^{2} \mathrm{~d} t+\mathcal{Q}(R)(b-a)
$$

and by conditions $\left(M_{1}\right),\left(M_{5}\right)$,

$$
\begin{aligned}
& 4 \int_{a}^{b} \frac{2}{\kappa(t)} \int_{0}^{\infty} \mu_{t}(s)\left\langle A^{1 / 2} \eta^{t}(s), \gamma(t)\right\rangle \mathrm{d} s \mathrm{~d} t \\
\leq & 8 \int_{a}^{b} \frac{1}{\kappa(t)}\|\gamma(t)\|_{-1}\left(\int_{0}^{\infty} \mu_{t}(s)\left\|\eta^{t}(s)\right\|_{2} \mathrm{~d} s\right) \mathrm{d} t \\
\leq & \mathcal{Q}(R)(b-a)+\mathcal{Q}(R) \int_{a}^{b} \kappa(t)\left\|\eta^{t}\right\|_{\mathcal{M}_{t}^{1 / 3}}^{2} \mathrm{~d} t .
\end{aligned}
$$

Exploiting (4.6)-(4.7) (taking $\varpi=\frac{1}{20}$ there) and making use of above two estimates, we obtain

$$
\begin{aligned}
& \Phi(b)+4 \Psi(b)+\int_{a}^{b} \Lambda_{1}(t) \mathrm{d} t+\frac{1}{5} \int_{a}^{b}\|u(t)\|_{2}^{2} \mathrm{~d} t \\
\leq & \Phi(a)+4 \Psi(a)-4 M \int_{a}^{b} \int_{0}^{\infty}\left[\partial_{t} \mu_{t}(s)+\partial_{s} \mu_{t}(s)\right]\left\|\eta^{t}(s)\right\|_{2}^{2} \mathrm{~d} s \mathrm{~d} t \\
& +\mathcal{Q}(R) \int_{a}^{b} \kappa(t)\left\|\eta^{t}\right\|_{\mathcal{M}_{t}^{1}}^{2} \mathrm{~d} t+\mathcal{Q}(R)(b-a) .
\end{aligned}
$$


EXPONENTIAL ATTRACTOR FOR THE VISCOELASTIC WAVE MODEL WITH TIME-DEPENDENT MEMORY KERNELS ${ }^{1} \mathrm{l}_{2}$

Taking $\alpha=\frac{2 \epsilon}{5}$, the combination of (4.39) and (4.40) gives

$$
\Lambda_{1}(b)+2 \epsilon \int_{a}^{b} \Lambda_{1}(t) \mathrm{d} t+\mathcal{J}_{1} \leq \Lambda_{1}(a)+\frac{\mathcal{Q}(R)}{\epsilon}(b-a),
$$

where we have used condition $\left(M_{4}\right)$ and the fact that

$$
\begin{aligned}
\mathcal{J}_{1} & =-(1-8 \epsilon M) \int_{a}^{b} \int_{0}^{\infty}\left[\partial_{t} \mu_{t}(s)+\partial_{s} \mu_{t}(s)\right]\left\|\eta^{t}(s)\right\|_{2}^{2} \mathrm{~d} s \mathrm{~d} t-2 \epsilon \mathcal{Q}(R) \int_{a}^{b} \kappa(t)\left\|\eta^{t}\right\|_{\mathcal{M}_{t}^{1}}^{2} \mathrm{~d} t \\
& \geq(\delta(1-8 \epsilon M)-2 \epsilon \mathcal{Q}(R)) \int_{a}^{b} \kappa(t)\left\|\eta^{t}\right\|_{\mathcal{M}_{t}^{1}}^{2} \mathrm{~d} t \geq 0
\end{aligned}
$$

for $\epsilon>0$ sufficiently small. Applying Lemma 4.1 (with $q_{1}=0, q_{2}=\frac{\mathcal{Q}(R)}{\epsilon}$ there) to (4.41) and making use of (4.38) give the conclusion of Lemma 4.9.

Lemma 4.10. Let Assumption 3.1 be valid. Then for any $z_{1 \tau}, z_{2 \tau} \in \mathcal{H}_{\tau}^{1}$ with $\left\|z_{i \tau}\right\|_{\mathcal{H}_{\tau}^{1}} \leq R, i=1,2$,

$$
\left\|U(t, \tau) z_{1 \tau}-U(t, \tau) z_{2 \tau}\right\|_{\mathcal{H}_{t}}^{2} \leq C e^{-\kappa(t-\tau)}\left\|z_{1 \tau}-z_{2 \tau}\right\|_{\mathcal{H}_{\tau}}^{2}+\mathcal{Q}(R) e^{t-\tau} \int_{\tau}^{t}\|\bar{u}(s)\|^{2} \mathrm{~d} s,
$$

where $C$ and $\kappa$ are positive constants independent of $R$, and

$$
\bar{z}(t)=\left(\bar{u}(t), \partial_{t} \bar{u}(t), \bar{\eta}^{t}\right)=z_{1}(t)-z_{2}(t) \text { and } z_{i}(t)=\left(u_{i}(t), \partial_{t} u_{i}(t), \eta_{i}^{t}\right)=U(t, \tau) z_{i \tau}, i=1,2 .
$$

Remark 4.11. Lemma 4.10 implies that the process $U(t, \tau): \mathbb{B}_{\tau}^{1}(R) \subset \mathcal{H}_{\tau} \rightarrow \mathcal{H}_{t}$ is quasi-stable for all $R>0$.

Proof of Lemma 4.10. We still split the solution $U(t, \tau) z_{i \tau}$ into the sum

$$
\begin{aligned}
U(t, \tau) z_{i \tau} & =U_{0}(t, \tau) z_{i \tau}+U_{1}(t, \tau) z_{i \tau} \\
& =\left(v_{i}(t), \partial_{t} v_{i}(t), \xi_{i}^{t}\right)+\left(w_{i}(t), \partial_{t} w_{i}(t), \zeta_{i}^{t}\right), \quad i=1,2,
\end{aligned}
$$

where $U_{0}(t, \tau) z_{i \tau}$ and $U_{1}(t, \tau) z_{i \tau}$ solves problem (4.11) and (4.12) (with $f_{0}=0$ and $f_{1}=f$ there), respectively. Then $\bar{v}=v_{1}-v_{2}$ solves

$$
\left\{\begin{array}{l}
\partial_{t t} \bar{v}+A \bar{v}+\int_{0}^{\infty} \mu_{t}(s) A \bar{\xi}^{t}(s) \mathrm{d} s=0, t>\tau \\
\left(\bar{v}(\tau), \partial_{t} \bar{v}(\tau), \bar{\xi}^{\tau}\right)=z_{1 \tau}-z_{2 \tau}
\end{array}\right.
$$

with

$$
\bar{\xi}^{t}(s)= \begin{cases}\bar{v}(t)-\bar{v}(t-s), & s \leq t-\tau, \\ \bar{\xi}_{\tau}(s-t+\tau)+\bar{v}(t)-\bar{v}_{\tau}, & s>t-\tau .\end{cases}
$$

And $\bar{w}=w_{1}-w_{2}$ solves

$$
\left\{\begin{array}{l}
\partial_{t t} \bar{w}+A \bar{w}+\int_{0}^{\infty} \mu_{t}(s) A \bar{\zeta}^{t}(s) \mathrm{d} s+f\left(u_{1}\right)-f\left(u_{2}\right)=0, \quad t>\tau \\
\left(\bar{w}(\tau), \partial_{t} \bar{w}(\tau), \bar{\zeta}^{\tau}\right)=0
\end{array}\right.
$$

with

$$
\bar{\zeta}^{t}(s)= \begin{cases}\bar{w}(t)-\bar{w}(t-s), & s \leq t-\tau \\ \bar{\zeta}_{\tau}(s-t+\tau)+\bar{w}(t)-\bar{w}_{\tau}, & s>t-\tau\end{cases}
$$


It follows from Lemmas 4.8 and 4.9 that

$$
\left\|z_{i}(t)\right\|_{\mathcal{H}_{t}^{1}}+\left\|U_{0}(t, \tau) z_{i \tau}\right\|_{\mathcal{H}_{t}^{1}}+\left\|U_{1}(t, \tau) z_{i \tau}\right\|_{\mathcal{H}_{t}^{1}} \leq \mathcal{Q}(R), \quad t \geq \tau
$$

Hence we can take the multiplier $2 \partial_{t} \bar{v}$ in Eq. (4.42) and obtain

$$
\|\bar{v}(b)\|_{1}^{2}+\left\|\partial_{t} \bar{v}(b)\right\|^{2}+2 \int_{a}^{b}\left\langle\bar{\xi}^{t}, \partial_{t} \bar{v}(t)\right\rangle_{\mathcal{M}_{t}} \mathrm{~d} t=\|\bar{v}(a)\|_{1}^{2}+\left\|\partial_{t} \bar{v}(a)\right\|^{2}, \forall b \geq a \geq \tau .
$$

By Lemma 4.2 (taking $\sigma=0$ there),

$$
\left\|\bar{\xi}^{b}\right\|_{\mathcal{M}_{b}}^{2}-\int_{a}^{b} \int_{0}^{\infty}\left[\partial_{t} \mu_{t}(s)+\partial_{s} \mu_{t}(s)\right]\left\|\bar{\xi}^{t}(s)\right\|_{1}^{2} \mathrm{~d} s \mathrm{~d} t \leq\left\|\bar{\xi}^{a}\right\|_{\mathcal{M}_{a}}^{2}+2 \int_{a}^{b}\left\langle\partial_{t} \bar{v}(t), \bar{\xi}^{t}\right\rangle_{\mathcal{M}_{t}} \mathrm{~d} t
$$

The combination of (4.45) and (4.46) gives

$$
\begin{aligned}
& \left\|\left(\bar{v}(b), \partial_{t} \bar{v}(b), \bar{\xi}^{b}\right)\right\|_{\mathcal{H}_{b}}^{2}-\int_{a}^{b} \int_{0}^{\infty}\left[\partial_{t} \mu_{t}(s)+\partial_{s} \mu_{t}(s)\right]\left\|\bar{\xi}^{t}(s)\right\|_{1}^{2} \mathrm{~d} s \mathrm{~d} t \\
\leq & \left\|\left(\bar{v}(a), \partial_{t} \bar{v}(a), \bar{\xi}^{a}\right)\right\|_{\mathcal{H}_{a}}^{2}, \quad \forall b \geq a \geq \tau .
\end{aligned}
$$

In order to obtain the sufficient regularity of the solutions needed for applying Lemma 4.3, we use the following approximating technique. We denote by

$$
\left(v_{\text {in }}(t), \partial_{t} v_{\text {in }}(t), \xi_{\text {in }}^{t}\right), \quad i=1,2
$$

the Galerkin approximations of $\left(v_{i}(t), \partial_{t} v_{i}(t), \xi_{i}^{t}\right), i=1,2$, with initial data

$$
\left(v_{i n}(\tau), \partial_{t} v_{i n}(\tau), \xi_{\text {in }}^{\tau}\right) \rightarrow z_{i \tau} \text { in } \mathcal{H}_{\tau}, \quad i=1,2
$$

It follows from (4.47)-(4.48) and condition $\left(M_{4}\right)$ that

$$
\begin{aligned}
& \lim _{n \rightarrow \infty}\left\|\left(v_{i n}(t), \partial_{t} v_{i n}(t), \xi_{i n}^{t}\right)-\left(v_{i}(t), \partial_{t} v_{i}(t), \xi_{i}^{t}\right)\right\|_{\mathcal{H}_{t}} \\
\leq & \lim _{n \rightarrow \infty}\left\|\left(v_{i n}(\tau), \partial_{t} v_{i n}(\tau), \xi_{i n}^{\tau}\right)-z_{i \tau}\right\|_{\mathcal{H}_{\tau}}=0, \quad \forall t \geq \tau, i=1,2 .
\end{aligned}
$$

For every $n \in \mathbb{N}$, let $\bar{v}_{n}=v_{1 n}-v_{2 n}, \bar{\xi}_{\tau n}=\xi_{1 n}^{\tau}-\xi_{\text {in }}^{\tau}$ and

$$
\bar{\xi}_{n}^{t}(s)= \begin{cases}\bar{v}_{n}(t)-\bar{v}_{n}(t-s), & s \leq t-\tau, \\ \bar{\xi}_{\tau n}(s-t+\tau)+\bar{v}_{n}(t)-\bar{v}_{\tau n}, & s>t-\tau .\end{cases}
$$

Obviously, formula (4.47) holds for all $\left(\bar{v}_{n}, \partial_{t} \bar{v}_{n}, \bar{\xi}_{n}^{t}\right), n \in \mathbb{N}$.

For every $\epsilon \in(0,1]$, we introduce the functional

$$
\Lambda_{v}^{n}(t)=\left\|\left(\bar{v}_{n}(t), \partial_{t} \bar{v}_{n}(t), \bar{\xi}_{n}^{t}\right)\right\|_{\mathcal{H}_{t}}^{2}+2 \epsilon\left[\Phi_{n}(t)+4 \Psi_{n}(t)\right], \quad n \in \mathbb{N},
$$

where the functionals $\Phi_{n}$ and $\Psi_{n}$ are defined by formulas (4.4)-(4.5), with

$$
\left(p(t), \partial_{t} p(t), \psi^{t}\right)=\left(\bar{v}_{n}(t), \partial_{t} \bar{v}_{n}(t), \bar{\xi}_{n}^{t}\right) \text { and } \gamma(t)=0 \text { in (4.1). }
$$

Thus, it follows from formula (4.8) that

$$
\frac{1}{2}\left\|\left(\bar{v}_{n}(t), \partial_{t} \bar{v}_{n}(t), \bar{\xi}_{n}^{t}\right)\right\|_{\mathcal{H}_{t}}^{2} \leq \Lambda_{v}^{n}(t) \leq \frac{3}{2}\left\|\left(\bar{v}_{n}(t), \partial_{t} \bar{v}_{n}(t), \bar{\xi}_{n}^{t}\right)\right\|_{\mathcal{H}_{t}}^{2}, \quad n \in \mathbb{N}
$$


EXPONENTIAL ATTRACTOR FOR THE VISCOELASTIC WAVE MODEL WITH TIME-DEPENDENT MEMORY KERNELS ${ }^{12} 25$

for $\epsilon>0$ sufficiently small. And by Lemma 4.3 (taking $\varpi=1 / 20$ and $\gamma(t)=0$ there) and a simple calculation we obtain

$$
\begin{aligned}
& \Phi_{n}(b)+4 \Psi_{n}(b)+\frac{7}{4} \int_{a}^{b}\left\|\bar{v}_{n}(t)\right\|_{1}^{2} \mathrm{~d} t+2 \int_{a}^{b}\left\|\partial_{t} \bar{v}_{n}(t)\right\|^{2} \mathrm{~d} t \\
\leq & \Phi_{n}(a)+4 \Psi_{n}(a)-4 M \int_{a}^{b} \int_{0}^{\infty}\left[\partial_{t} \mu_{t}(s)+\partial_{s} \mu_{t}(s)\right]\left\|\bar{\xi}_{n}^{t}(s)\right\|_{1}^{2} \mathrm{~d} s \mathrm{~d} t+C \int_{a}^{b} \kappa(t)\left\|\bar{\xi}_{n}^{t}\right\|_{\mathcal{M}_{t}}^{2} \mathrm{~d} t .
\end{aligned}
$$

The combination of (4.47) and (4.51) gives

$$
\Lambda_{v}^{n}(b)+2 \epsilon \int_{a}^{b} \Lambda_{v}^{n}(t) \mathrm{d} t \leq \Lambda_{v}^{n}(a)
$$

for $\epsilon>0$ suitably small, where we have used condition $\left(M_{4}\right)$. Hence applying Lemma 4.1, with $q_{1}=q_{2}=0$ there, and making use of (4.50), we obtain

$$
\left\|\left(\bar{v}_{n}(t), \partial_{t} \bar{v}_{n}(t), \bar{\xi}_{n}^{t}\right)\right\|_{\mathcal{H}_{t}}^{2} \leq 3 e^{-\epsilon(t-\tau)}\left\|\left(\bar{v}_{n}(\tau), \partial_{t} \bar{v}_{n}(\tau), \bar{\xi}_{n}^{\tau}\right)\right\|_{\mathcal{H}_{\tau}}^{2}, \quad \forall t \geq \tau, n \in \mathbb{N} .
$$

Thus, by (4.48)-(4.49) and formula (4.52), we have

$$
\begin{aligned}
\left\|\left(\bar{v}(t), \partial_{t} \bar{v}(t), \bar{\xi}^{t}\right)\right\|_{\mathcal{H}_{t}}^{2} & =\lim _{n \rightarrow \infty}\left\|\left(\bar{v}_{n}(t), \partial_{t} \bar{v}_{n}(t), \bar{\xi}_{n}^{t}\right)\right\|_{\mathcal{H}_{t}}^{2} \\
& \leq 3 e^{-\epsilon(t-\tau)} \lim _{n \rightarrow \infty}\left\|\left(\bar{v}_{n}(\tau), \partial_{t} \bar{v}_{n}(\tau), \bar{\xi}_{n}^{\tau}\right)\right\|_{\mathcal{H}_{\tau}}^{2} \\
& =3 e^{-\epsilon(t-\tau)}\left\|z_{1 \tau}-z_{2 \tau}\right\|_{\mathcal{H}_{\tau}}^{2} .
\end{aligned}
$$

Taking into account estimate (4.44), we can use multiplier $2 \partial_{t} \bar{w}$ in Eq. (4.43) and arrive at

$$
\begin{aligned}
& \frac{\mathrm{d}}{\mathrm{d} t}\left[\|\bar{w}(t)\|_{1}^{2}+\left\|\partial_{t} \bar{w}(t)\right\|^{2}\right]+2\left\langle\bar{\zeta}^{t}, \partial_{t} \bar{w}(t)\right\rangle_{\mathcal{M}_{t}} \\
= & 2\left\langle f\left(u_{2}\right)-f\left(u_{1}\right), \partial_{t} \bar{w}(t)\right\rangle \\
\leq & C\left(1+\left\|u_{1}(t)\right\|_{L^{\infty}}^{2}+\left\|u_{2}(t)\right\|_{L^{\infty}}^{2}\right)\|\bar{u}(t)\|\left\|\partial_{t} \bar{w}(t)\right\| \\
\leq & \mathcal{Q}(R)\|\bar{u}(t)\|^{2}+\left\|\partial_{t} \bar{w}(t)\right\|^{2},
\end{aligned}
$$

which implies that

$$
\|\bar{w}(t)\|_{1}^{2}+\left\|\partial_{t} \bar{w}(t)\right\|^{2}+2 \int_{\tau}^{t}\left\langle\bar{\zeta}^{s}, \partial_{s} \bar{w}(s)\right\rangle_{\mathcal{M}_{s}} d s \leq \mathcal{Q}(R) \int_{\tau}^{t}\left(\|\bar{u}(s)\|^{2}+\left\|\partial_{t} \bar{w}(s)\right\|^{2}\right) d s, \quad \forall t \geq \tau,
$$

where we have used condition (3.1), estimate (4.44) and the Sobolev embedding $H^{2} \hookrightarrow L^{\infty}$. Thus, making use of condition $\left(M_{4}\right)$ and Lemma 4.2 (with $\sigma=0$ there), we obtain

$$
\left\|\left(\bar{w}(t), \partial_{t} \bar{w}(t), \bar{\zeta}^{t}\right)\right\|_{\mathcal{H}_{t}}^{2} \leq \mathcal{Q}(R) \int_{\tau}^{t}\|\bar{u}(s)\|^{2} \mathrm{~d} s+\int_{\tau}^{t}\left\|\left(\bar{w}(s), \partial_{t} \bar{w}(s), \bar{\zeta}^{s}\right)\right\|_{\mathcal{H}_{s}}^{2} \mathrm{~d} s .
$$

Applying the Gronwall inequality to above estimate gives

$$
\left\|\left(\bar{w}(t), \partial_{t} \bar{w}(t), \bar{\zeta}^{t}\right)\right\|_{\mathcal{H}_{t}}^{2} \leq \mathcal{Q}(R) e^{t-\tau} \int_{\tau}^{t}\|\bar{u}(s)\|^{2} \mathrm{~d} s, \quad \forall t \geq \tau .
$$


The combination of (4.53) and (4.54) yields

$$
\begin{aligned}
& \left\|U(t, \tau) z_{1 \tau}-U(t, \tau) z_{2 \tau}\right\|_{\mathcal{H}_{t}}^{2} \\
\leq & C\left[\left\|\left(\bar{v}(t), \partial_{t} \bar{v}(t), \bar{\xi}^{t}\right)\right\|_{\mathcal{H}_{t}}^{2}+\left\|\left(\bar{w}(t), \partial_{t} \bar{w}(t), \bar{\zeta}^{t}\right)\right\|_{\mathcal{H}_{t}}^{2}\right] \\
\leq & C e^{-\epsilon(t-\tau)}\left\|z_{1 \tau}-z_{2 \tau}\right\|_{\mathcal{H}_{\tau}}^{2}+\mathcal{Q}(R) e^{t-\tau} \int_{\tau}^{t}\|\bar{u}(s)\|^{2} \mathrm{~d} s, \quad t \geq \tau,
\end{aligned}
$$

which completes the proof.

\section{PROOF OF THE MAIN RESUlT}

The purpose of this section is to prove Theorem 3.5 by applying the abstract criteria obtained in Section 2. This argument is challenging because of the hyperbolicity of the problem which results in without any additional regularity of its solutions, so we put forward a new technique to overcome this difficulty. To this end, we first establish a specially pullback attracting family.

Lemma 5.1. Let Assumption 3.1 be valid. Then there exists a family $\{B(t)\}_{t \in \mathbb{R}}$, with $B(t) \subset \mathcal{H}_{t}$ for each $t \in \mathbb{R}$, possessing the following properties:

: (i) for every $t \in \mathbb{R}$, the section $B(t)$ is closed in $\mathcal{H}_{t}$ and

$$
B(t) \subset \mathbb{B}_{t}\left(\mathcal{R}_{0}\right) \cap \mathbb{B}_{t}^{1}(\mathcal{R})
$$

for some constants $\mathcal{R}>0$ and $\mathcal{R}_{0}>R_{1}$, where $R_{1}$ is given by Lemma 4.5;

: (ii) there exist positive constants $\kappa$ and $\tau_{1}$ such that

$$
\operatorname{dist}_{\mathcal{H}_{t}}\left(U(t, \tau) \mathbb{B}_{\tau}\left(R_{1}\right), B(t)\right) \leq \mathcal{Q}\left(R_{1}\right) e^{-\kappa(t-\tau)}, \forall \tau \leq t-\tau_{1}, t \in \mathbb{R} ;
$$

: (iii) there exists a positive constant $T_{1}$ such that

$$
U(t, \tau) B(\tau) \subset B(t), \quad \forall \tau \leq t-T_{1}, t \in \mathbb{R} .
$$

Proof. For any $\tau \in \mathbb{R}$ and $z_{\tau} \in \mathbb{B}_{\tau}\left(R_{1}\right)$, it follows from Lemma 4.6 that

$$
\left\|U_{0}(t, \tau) z_{\tau}\right\|_{\mathcal{H}_{t}}^{2} \leq \mathcal{Q}\left(R_{1}\right) e^{-\omega(t-\tau)} \text { and }\left\|U_{1}(t, \tau) z_{\tau}\right\|_{\mathcal{H}_{t}^{1 / 3}}^{2} \leq \mathcal{Q}\left(R_{1}\right), \quad \forall t \geq \tau,
$$

which implies that there exists a positive constant $\mathcal{R}_{1}$ depending only on $R_{1}$ such that

$$
\operatorname{dist}_{\mathcal{H}_{t}}\left(U(t, \tau) \mathbb{B}_{\tau}\left(R_{1}\right), \mathbb{B}_{t}^{1 / 3}\left(\mathcal{R}_{1}\right)\right) \leq \mathcal{Q}\left(R_{1}\right) e^{-\omega(t-\tau)}, \forall t \geq \tau .
$$

Similarly, we infer from Lemma 4.8 that, for any $z_{\tau} \in \mathbb{B}_{\tau}^{1 / 3}\left(\mathcal{R}_{1}\right)$,

$$
\left\|U_{0}(t, \tau) z_{\tau}\right\|_{\mathcal{H}_{t}}^{2} \leq \mathcal{Q}\left(\mathcal{R}_{1}\right) e^{-\omega(t-\tau)} \text { and }\left\|U_{1}(t, \tau) z_{\tau}\right\|_{\mathcal{H}_{t}^{1}}^{2} \leq \mathcal{Q}\left(\mathcal{R}_{1}\right), \quad t \geq \tau .
$$

Since $\mathcal{R}_{1}$ depends only on $R_{1}$, we can find a constant $\mathcal{R}_{2}$ depending only on $R_{1}$ such that

$$
\operatorname{dist}_{\mathcal{H}_{t}}\left(U(t, \tau) \mathbb{B}_{\tau}^{1 / 3}\left(\mathcal{R}_{1}\right), \mathbb{B}_{t}^{1}\left(\mathcal{R}_{2}\right)\right) \leq \mathcal{Q}\left(R_{1}\right) e^{-\omega(t-\tau)}, \quad \forall t \geq \tau .
$$

It follows from Definition 2.2 and Lemma 4.5 that there exists a positive constant $e\left(R_{1}\right)$ depending only on $R_{1}$ such that

$$
U(t, \tau) \mathbb{B}_{\tau}\left(R_{1}\right) \subset \mathbb{B}_{t}\left(R_{1}\right), \quad \forall \tau \leq t-e\left(R_{1}\right)
$$


EXPONENTIAL ATTRACTOR FOR THE VISCOELASTIC WAVE MODEL WITH TIME-DEPENDENT MEMORY KERNELS $1 \frac{1}{27}$

Let $\theta=\frac{\omega}{\mathcal{Q}\left(R_{1}\right)+2 \omega}$. Obviously,

$$
\theta \in(0,1) \text { and }-\omega \theta=-\omega+\left(\mathcal{Q}\left(R_{1}\right)+\omega\right) \theta .
$$

We infer from formula (5.6) that

$$
U((1-\theta) t+\theta \tau, \tau) \mathbb{B}_{\tau}\left(R_{1}\right) \subset \mathbb{B}_{(1-\theta) t+\theta \tau}\left(R_{1}\right), \quad \forall \tau \leq t-e_{1},
$$

where $e_{1}=\frac{e\left(R_{1}\right)}{1-\theta}>0$. Thus, it follows from Theorem 3.3 and formula (5.4)-(5.7) that

$$
\begin{aligned}
& \operatorname{dist}_{\mathcal{H}_{t}}\left(U(t, \tau) \mathbb{B}_{\tau}\left(R_{1}\right), \mathbb{B}_{t}^{1}\left(\mathcal{R}_{2}\right)\right) \\
\leq & \operatorname{dist}_{\mathcal{H}_{t}}\left(U(t,(1-\theta) t+\theta \tau) U((1-\theta) t+\theta \tau, \tau) \mathbb{B}_{\tau}\left(R_{1}\right), U(t,(1-\theta) t+\theta \tau) \mathbb{B}_{(1-\theta) t+\theta \tau}^{1 / 3}\left(\mathcal{R}_{1}\right)\right) \\
& +\operatorname{dist}_{\mathcal{H}_{t}}\left(U(t,(1-\theta) t+\theta \tau) \mathbb{B}_{(1-\theta) t+\theta \tau}^{1 / 3}\left(\mathcal{R}_{1}\right), \mathbb{B}_{t}^{1}\left(\mathcal{R}_{2}\right)\right) \\
\leq & \mathcal{Q}\left(R_{1}\right) \exp \left\{\mathcal{Q}\left(R_{1}\right) \theta(t-\tau)\right\} \operatorname{dist}_{\mathcal{H}}(1-\theta) t+\theta \tau \\
& +\mathcal{Q}\left(R_{1}\right) e^{-\omega \theta(t-\tau)} \\
\leq & \mathcal{Q}\left(R_{1}\right) \exp \left\{[-\omega+(1-\theta) t+\theta \tau, \tau) \mathbb{B}_{\tau}\left(R_{1}\right), \mathbb{B}_{(1-\theta) t+\theta \tau}^{1 / 3}\left(\mathcal{R}_{1}\right)\right) \\
\leq & \mathcal{Q}\left(R_{1}\right) e^{-\omega \theta(t-\tau)}, \forall \tau \leq t-e_{1} .
\end{aligned}
$$

For every $z \in \mathbb{B}_{t}^{1}\left(\mathcal{R}_{2}\right)$,

$$
\|z\|_{\mathcal{H}_{t}} \leq \lambda_{1}^{-1 / 2}\|z\|_{\mathcal{H}_{t}^{1}} \leq \lambda_{1}^{-1 / 2} \mathcal{R}_{2}, \quad \forall t \in \mathbb{R}
$$

which implies

$$
\mathbb{B}_{t}^{1}\left(\mathcal{R}_{2}\right) \subset \mathbb{B}_{t}\left(\lambda_{1}^{-1 / 2} \mathcal{R}_{2}\right) \subset \mathbb{B}_{t}\left(\mathcal{R}_{3}\right) \text { and } \mathbb{B}_{t}\left(R_{1}\right) \subset \mathbb{B}_{t}\left(\mathcal{R}_{3}\right), \quad \forall t \in \mathbb{R},
$$

where $\mathcal{R}_{3}=R_{1}+\lambda_{1}^{-1 / 2} \mathcal{R}_{2}$ depends only on $R_{1}$. By Lemma 4.5 and formula (5.9), there exists a constant $e_{2}>0$ such that

$$
U(t, \tau) \mathbb{B}_{\tau}\left(\mathcal{R}_{3}\right) \subset \mathbb{B}_{t}\left(R_{1}\right) \subset \mathbb{B}_{t}\left(\mathcal{R}_{3}\right), \forall \tau \leq t-e_{2} .
$$

It follows from Lemma 4.7 that for any $z_{\tau} \in \mathbb{B}_{\tau}\left(\mathcal{R}_{3}\right) \cap \mathcal{H}_{\tau}^{1 / 3}$,

$$
\left\|U(t, \tau) z_{\tau}\right\|_{\mathcal{H}_{t}^{1 / 3}}^{2} \leq \mathcal{Q}\left(\mathcal{R}_{3}+\left\|z_{\tau}\right\|_{\mathcal{H}_{\tau}^{1 / 3}}\right) e^{-\omega(t-\tau)}+\mathcal{R}_{4}, \quad \forall t \geq \tau
$$

where the positive constant $\mathcal{R}_{4}$ depends only on $R_{1}$.

Similarly, for every $z \in \mathbb{B}_{t}^{1}\left(\mathcal{R}_{2}\right)$, we have

$$
\|z\|_{\mathcal{H}_{t}^{1 / 3}} \leq \lambda_{1}^{-1 / 3}\|z\|_{\mathcal{H}_{t}^{1}} \leq \lambda_{1}^{-1 / 3} \mathcal{R}_{2}, \quad \forall t \in \mathbb{R},
$$

which implies

$$
\mathbb{B}_{t}^{1}\left(\mathcal{R}_{2}\right) \subset \mathbb{B}_{t}^{1 / 3}\left(\lambda_{1}^{-1 / 3} \mathcal{R}_{2}\right) \subset \mathbb{B}_{t}^{1 / 3}\left(\mathcal{R}_{5}\right), \quad \forall t \in \mathbb{R},
$$

where $\mathcal{R}_{5}=\mathcal{R}_{4}+\lambda_{1}^{-1 / 3} \mathcal{R}_{2}$ depends only on $R_{1}$. It follows from formula (5.11) that there exists a positive constant $e_{3}$ such that

$$
U(t, \tau)\left[\mathbb{B}_{\tau}\left(\mathcal{R}_{3}\right) \cap \mathbb{B}_{\tau}^{1 / 3}\left(\mathcal{R}_{5}\right)\right] \subset \mathbb{B}_{t}^{1 / 3}\left(\mathcal{R}_{5}\right), \quad \forall \tau \leq t-e_{3} .
$$


Lemma 4.9 shows that for any $z_{\tau} \in \mathbb{B}_{\tau}^{1 / 3}\left(\mathcal{R}_{5}\right) \cap \mathcal{H}_{\tau}^{1}$,

$$
\left\|U(t, \tau) z_{\tau}\right\|_{\mathcal{H}_{t}^{1}}^{2} \leq \mathcal{Q}\left(\mathcal{R}_{5}+\left\|z_{\tau}\right\|_{\mathcal{H}_{\tau}^{1}}\right) e^{-\omega(t-\tau)}+\mathcal{R}_{6}, \quad \forall t \geq \tau,
$$

where the positive constant $\mathcal{R}_{6}$ depends only on $R_{1}$. Obviously,

$$
\mathbb{B}_{t}^{1}\left(\mathcal{R}_{2}\right) \subset \mathbb{B}_{t}^{1}\left(\mathcal{R}_{7}\right) \text { with } \mathcal{R}_{7}=\mathcal{R}_{2}+\mathcal{R}_{6}, \quad \forall t \in \mathbb{R} .
$$

Thus formula (5.14) implies that there is a positive constant $e_{4}$ such that

$$
U(t, \tau)\left[\mathbb{B}_{\tau}^{1 / 3}\left(\mathcal{R}_{5}\right) \cap \mathbb{B}_{\tau}^{1}\left(\mathcal{R}_{7}\right)\right] \subset \mathbb{B}_{t}^{1}\left(\mathcal{R}_{7}\right), \quad \forall \tau \leq t-e_{4} .
$$

Let

$$
B(t)=\mathbb{B}_{t}\left(\mathcal{R}_{3}\right) \cap \mathbb{B}_{t}^{1 / 3}\left(\mathcal{R}_{5}\right) \cap \mathbb{B}_{t}^{1}\left(\mathcal{R}_{7}\right), \quad \forall t \in \mathbb{R} .
$$

We show that $\{B(t)\}_{t \in \mathbb{R}}$ is the desired family.

(i) Obviously, for every $t \in \mathbb{R}, B(t)$ is closed in $\mathcal{H}_{t}$ and

$$
B(t) \subset \mathbb{B}_{t}\left(\mathcal{R}_{3}\right) \cap \mathbb{B}_{t}^{1}\left(\mathcal{R}_{7}\right),
$$

that is, conclusion (5.1) is valid, with $\mathcal{R}_{0}=\mathcal{R}_{3}>R_{1}$ and $\mathcal{R}=\mathcal{R}_{7}$.

(ii) It follows from formulas (5.9), (5.12) and (5.15) that $\mathbb{B}_{t}^{1}\left(\mathcal{R}_{2}\right) \subset B(t)$ holds for all $t \in \mathbb{R}$. Then we infer from estimates $(5.8)$ that

$$
\begin{aligned}
\operatorname{dist}_{\mathcal{H}_{t}}\left(U(t, \tau) \mathbb{B}_{\tau}\left(R_{1}\right), B(t)\right) & \leq \operatorname{dist}_{\mathcal{H}_{t}}\left(U(t, \tau) \mathbb{B}_{\tau}\left(R_{1}\right), \mathbb{B}_{t}^{1}\left(\mathcal{R}_{2}\right)\right) \\
& \leq \mathcal{Q}\left(R_{1}\right) e^{-\omega \theta(t-\tau)}, \quad \forall \tau \leq t-e_{1},
\end{aligned}
$$

that is, formula (5.2) holds, with $\kappa=\omega \theta$ and $\tau_{1}=e_{1}$.

(iii) Taking $T_{1}=\max \left\{e_{2}, e_{3}, e_{4}\right\}$ and making use of formulas (5.10), (5.13) and (5.16) yield

$$
\begin{aligned}
& U(t, \tau) B(\tau) \subset U(t, \tau) \mathbb{B}_{\tau}\left(\mathcal{R}_{3}\right) \subset \mathbb{B}_{t}\left(\mathcal{R}_{3}\right), \\
& U(t, \tau) B(\tau) \subset U(t, \tau)\left[\mathbb{B}_{\tau}\left(\mathcal{R}_{3}\right) \cap \mathbb{B}_{\tau}^{1 / 3}\left(\mathcal{R}_{5}\right)\right] \subset \mathbb{B}_{t}^{1 / 3}\left(\mathcal{R}_{5}\right), \\
& U(t, \tau) B(\tau) \subset U(t, \tau)\left[\mathbb{B}_{\tau}^{1 / 3}\left(\mathcal{R}_{5}\right) \cap \mathbb{B}_{\tau}^{1}\left(\mathcal{R}_{7}\right)\right] \subset \mathbb{B}_{t}^{1}\left(\mathcal{R}_{7}\right), \quad \forall \tau \leq t-T_{1}, t \in \mathbb{R} .
\end{aligned}
$$

Therefore,

$$
U(t, \tau) B(\tau) \subset \mathbb{B}_{t}\left(\mathcal{R}_{3}\right) \cap \mathbb{B}_{t}^{1 / 3}\left(\mathcal{R}_{5}\right) \cap \mathbb{B}_{t}^{1}\left(\mathcal{R}_{7}\right)=B(t), \quad \forall \tau \leq t-T_{1}, t \in \mathbb{R} .
$$

This completes the proof.

Proof of Theorem 3.5. It follows from Lemma 5.1 that the family $\{B(t)\}_{t \in \mathbb{R}}$ is uniformly bounded in $\left\{\mathcal{H}_{t}\right\}_{t \in \mathbb{R}}, B(t)$ is closed in $\mathcal{H}_{t}$ for each $t \in \mathbb{R}$, and there exists a positive constant $T>T_{1}$ such that $\eta^{2}=C e^{-\kappa T}<\frac{1}{4}$ and

$$
U(t, t-\tau) B(t-\tau) \subset B(t), \forall t \in \mathbb{R}, \tau \geq T,
$$

where $T_{1}$ is as shown in Lemma 5.1. It follows from formula (3.2) that for any $t \in \mathbb{R}$,

$$
\left\|U(t, t-\tau) z_{1}-U(t, t-\tau) z_{1}\right\|_{\mathcal{H}_{t}} \leq L_{1}\left\|z_{1}-z_{2}\right\|_{\mathcal{H}_{t-\tau}}, \quad \forall z_{1}, z_{2} \in \mathbb{B}_{t}\left(\mathcal{R}_{0}\right), \tau \in[0, T]
$$


EXPONENTIAL ATTRACTOR FOR THE VISCOELASTIC WAVE MODEL WITH TIME-DEPENDENT MEMORY KERNELS 1429

where the positive constant $L_{1}$ depends only on $\mathcal{R}_{0}$ and $T$.

Define the space

$$
Z=\left\{u \in L^{2}\left(0, T ; H^{1}\right) \mid \partial_{t} u \in L^{2}(0, T ; H)\right\}
$$

equipped with the norm

$$
\|u\|_{Z}=\left\|\left(u, \partial_{t} u\right)\right\|_{L^{2}\left(0, T ; H^{1} \times H\right)} .
$$

Obviously, $Z$ is a Banach space. And the functional

$$
n_{Z}(u)=\mathcal{Q}\left(\mathcal{R}_{0}+T\right)\|u\|_{L^{2}(0, T ; H)}
$$

is a compact semi-norm on $Z$ (cf. [25]). For any given $t \in \mathbb{R}$, we define the mapping

$$
K_{t}: B(t-T) \rightarrow Z, \quad K_{t} z=u(\cdot+t-T), \forall z \in B(t-T),
$$

where $u(\cdot+t-T)$ means $u(s+t-T), s \in[0, T]$, and

$$
\left(u(s+t-T), \partial_{t} u(s+t-T), \eta^{s+t-T}\right)=U(s+t-T, t-T) z .
$$

Lemma 4.10 shows that

$$
\left\|U(t, t-T) z_{1}-U(t, t-T) z_{2}\right\|_{\mathcal{H}_{t}} \leq \eta\left\|z_{1}-z_{2}\right\|_{\mathcal{H}_{t-T}}+n_{Z}\left(K_{t} z_{1}-K_{t} z_{2}\right),
$$

and we infer from formulas (3.2) and (5.1) that

$$
\begin{aligned}
\left\|K_{t} z_{1}-K_{t} z_{2}\right\|_{Z}^{2} & \leq \int_{0}^{T}\left\|U(s+t-T, t-T) z_{1}-U(s+t-T, t-T) z_{2}\right\|_{\mathcal{H}_{s-t+T}}^{2} \mathrm{~d} s \\
& \leq e^{\mathcal{Q}\left(\mathcal{R}_{0}\right) T}\left\|z_{1}-z_{2}\right\|_{\mathcal{H}_{t-T}}^{2}, \quad \forall z_{1}, z_{2} \in B(t-T), \quad t \in \mathbb{R} .
\end{aligned}
$$

Thus the family $\mathcal{B}=\{B(t)\}_{t \in \mathbb{R}}$ satisfies conditions $\left(H_{1}\right)-\left(H_{3}\right)$ of Theorem 2.5.

Moreover, by Lemma $4.5,\left\{\mathbb{B}_{t}\left(R_{1}\right)\right\}_{t \in \mathbb{R}}$ is a uniformly time-dependent absorbing set of the process $U(t, \tau)$. And formulas (5.1)-(5.2) and (5.17) show that $\left\{\mathbb{B}_{t}\left(R_{1}\right)\right\}_{t \in \mathbb{R}}$ satisfies the conditions of Corollary 2.7. Therefore, the process $U(t, \tau)$ has a time-dependent exponential attractor $\mathcal{E}=\{E(t)\}_{t \in \mathbb{R}}$, with $E(t) \subset B(t) \subset \mathbb{B}_{t}^{1}(\mathcal{R})$ for each $t \in \mathbb{R}$.

\section{REFERENCES}

[1] V. V. Chepyzhov, M. Conti, V. Pata, A minimal approach to the theory of global attractor, Discrete Contin. Dyn. Syst., 32 (2012) 2079-2088.

[2] I. Chueshov, I. Lasiecka, Attractors for second order evolution equations, J. Dynam. Diff. Eqs., 16 (2004) 469-512.

[3] I. Chueshov, I. Lasiecka, Long-time behavior of second order evolution equations with nonlinear damping, Memoirs of AMS 912, Amer. Math. Soc. Providence, 2008.

[4] I. Chueshov, Dynamics of Quasi-Stable Dissipative Systems, Springer, New York, 2015.

[5] R. M. Christensen, Theory of viscoelasticity: an introduction, Academic Press, New York, 1982.

[6] M. Conti, V. Pata, R. Temam, Attractors for the processes on time-dependent spaces. Application to wave equations, J. Differential Equations, 255 (2013) 1254-1277.

[7] M. Conti, V. Danese, C. Giorgi, V. Pata, A model of viscoelasticity with time-dependent memory kernels, Amer J Math., 140(2) (2018) 349-389.

[8] M. Conti, V. Danese, V. Pata, Viscoelasticity with time-dependent memory kernels, II: asymptotical behavior of solutions, Amer J Math., 140(6) (2018) 1687-1729. 
[9] V. Danese, P. G. Geredeli, V. Pata, Exponential attractors for abstract equations with memory and applications to viscoelasticity, Discrete Contin. Dyn. Syst., 35(7) (2015) 2881-2904.

[10] C. M. Dafermos, Asymptotic stability in viscoelasticity, Arch. Rational Mech. Anal., 37 (1970) 297-308.

[11] C. M. Dafermos, Contraction semigroups and trend to equilibrium in continuum mechanics, In "Applications of Methods of Functional Analysis to Problems in Mechanics" ( P. Germain and B. Nayroles, Eds.), pp. 295-306, Lecture Notes in Mathematics 503, Springer-Verlag, Berlin-New York, 1976.

[12] F. Dell'Oro, V. Pata, Long-term analysis of strongly damped nonlinear wave equations, Nonlinearity, 24 (2011) 3413-3435.

[13] A. Eden, C. Foias, B. Nicolaenko, R. Temam, Exponential attractors for dissipative evolution equations, Masson, Paris, 1994.

[14] M. Efendiev, A. Miranville, S. Zelik, Exponential attractors for a nonlinear reaction-diffusion system in $\mathbb{R}^{3}$, C. R. Acad. Sci. Paris Sér. I Math., 330 (2000) 713-718.

[15] M. Efendiev, A. Miranville, S. Zelik, Exponential attractors and finite-dimensional reduction for nonautonomous dynamical systems, Proc. Roy. Soc. Edinburgh Sect., A 13 (2005) 703-730.

[16] M. Fabrizio, B. Lazzari, On the existence and asymptotic stability of solutions for linear viscoelastic solids, Arch. Rational Mech. Anal., 116 (1991) 139-152.

[17] M. Fabrizio, A. Morro, Mathematical problems in linear viscoelasticity, SIAM Studies Appl. Math. 12, Philadelphia, PA, 1992.

[18] C. Giorgi, B. Lazzari, On the stability for linear viscoelastic solids, Quart. Appl. Math., 55 (1997) 659-675.

[19] V. K. Kalantarov, Attractors for some nonlinear problems of mathematical physics, J. Soviet Math., 40 (1988) 619-622.

[20] Z. Liu, S. Zheng, On the exponential stability of linear viscoelasticity and thermoviscoelasticity, Quart. Appl. Math., 54 (1996) 21-31.

[21] A. Miranville, S. Zelik, Attractors for dissipative partial differential equations in bounded and unbounded domains, in "Handbook of Differential Equations: Evolutionary Equations", Vol. 4 (C. M. Dafermos and M. Pokorny, Eds.), Elsevier, Amsterdam, 2008.

[22] J. E. Muñoz Rivera, Asymptotic behaviour in linear viscoelasticity, Quart. Appl. Math., 52 (1994) 629-648.

[23] F. Di Plinio, G. S.Duane, R. Temam, Time dependent attractor for the oscillon equation, Discrete Contin. Dyn. Syst., 29 (2011) 141-167.

[24] M. Renardy, W. J. Hrusa, J. A. Nohel, Mathematical problems in viscoelasticity, Longman Scientific \& Technical, Harlow John Wiley \& Sons, Inc., New York, 1987.

[25] J. Simon, Compact sets in the space $L^{p}(0, T ; B)$, Ann. Mat. Pura Appl., 146 (1986) 65-96.

[26] Z. J. Yang, Y. N. Li, Criteria on the existence and stability of pullback exponential attractors and their application to non-autonomous Kirchhoff wave models, Discrete Contin. Dyn. Syst., 38 (2018) 2629-2653. 